Review

\title{
Breastfeeding and depression: A systematic review of the literature
}

\author{
Cláudia Castro Dias*, Bárbara Figueiredo \\ School of Psychology, University of Minho, Campus de Gualtar, 4710-057 Braga, Portugal
}

\section{A R T I C L E I N F O}

\section{Article history:}

Received 18 March 2014

Received in revised form

11 September 2014

Accepted 14 September 2014

Available online 28 September 2014

\section{Keywords:}

Breastfeeding

Bottle-feeding

Postpartum depression

Pregnancy depression

\begin{abstract}
A B S T R A C T
Background: Research has separately indicated associations between pregnancy depression and breastfeeding, breastfeeding and postpartum depression, and pregnancy and postpartum depression. This paper aimed to provide a systematic literature review on breastfeeding and depression, considering both pregnancy and postpartum depression.

Methods: An electronic search in three databases was performed using the keywords: "breast feeding", "bottle feeding”, “depression", “pregnancy", and "postpartum”. Two investigators independently evaluated the titles and abstracts in a first stage and the full-text in a second stage review. Papers not addressing the association among breastfeeding and pregnancy or postpartum depression, non-original research and research focused on the effect of anti-depressants were excluded. 48 studies were selected and included. Data were independently extracted.

Results: Pregnancy depression predicts a shorter breastfeeding duration, but not breastfeeding intention or initiation. Breastfeeding duration is associated with postpartum depression in almost all studies. Postpartum depression predicts and is predicted by breastfeeding cessation in several studies. Pregnancy and postpartum depression are associated with shorter breastfeeding duration. Breastfeeding may mediate the association between pregnancy and postpartum depression. Pregnancy depression predicts shorter breastfeeding duration and that may increase depressive symptoms during postpartum. Limitations: The selected keywords may have led to the exclusion of relevant references.

Conclusions: Although strong empirical evidence regarding the associations among breastfeeding and pregnancy or postpartum depression was separately provided, further research, such as prospective studies, is needed to clarify the association among these three variables. Help for depressed pregnant women should be delivered to enhance both breastfeeding and postpartum psychological adjustment.
\end{abstract}

(c) 2014 Elsevier B.V. All rights reserved.

\section{Contents}

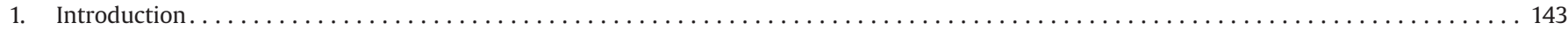

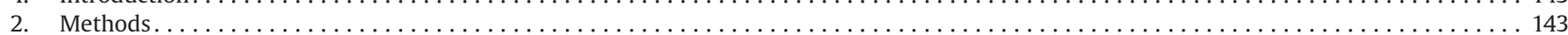

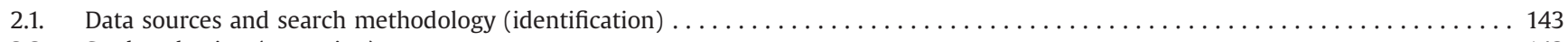

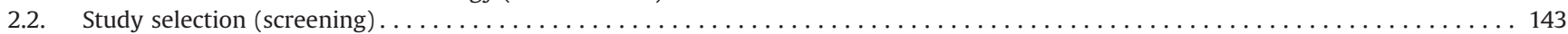

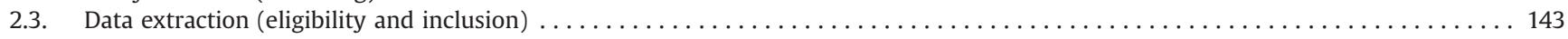

\footnotetext{
Abbreviations: AAS, Adult Attachment Scale; AKUADS, Aga Khan University Anxiety and Depression Scale; BAI, Beck Anxiety Inventory; BDI, Beck Depression Inventory; BDI-II, Beck Depression Inventory-Second Edition; BSES, Breastfeeding Self-Efficacy Scale; BSES-SF, Breastfeeding Self-Efficacy Scale-Short Form; BSSS, Berlin Social Support Scales; CERQ Cognitive Emotion Regulation Questionnaire; CES-D, Center for Epidemiologic Studies Depression Scale; CGI, Clinical Global Impressions Scale; DIS, Diagnostic Interview Schedule; EMQ/EFQ Experience of Motherhood/Fatherhood Questionnaire; EPDS, Edinburgh Postpartum Depression Scale; GAMS, General Adjustment and Morale Scale; GASD, Goldberg Scales of Anxiety and Depression; HADS, Hospital Anxiety and Depression Scale; HDRS, Hamilton Depression Rating Scale; HRS, Health Responses Scale; IDS, Inventory of Depressive Symptomatology; IFQ Infant Feeding Questionnaire; LIFE, Longitudinal Interval Follow-Up Evaluation; MABS, Mother and Baby Scale; MINI, Mini International Neuropsychiatric Interview; MOS, Medical Outcomes Study; MSPSS, Multidimensional Scale of Perceived Social Support; QLDS, Quality of Life in Depression Scale; PDSS, Postpartum Depression Screening Scale; PSE, Present State Examination; PSI/SF, Parenting Stress Index, Short Form; PSS, Perceived Stress Scale; RSES, Rosenberg Self Esteem Scale; SAS, Zung Self-Rating Anxiety Scale; SCID, Structured Clinical Interview for DSM-IV; SCL - 8, Hopkins Symptom Checklist; SDS, Zung SelfRating Depression Scale; SES, Socio-Economic Status Scale; SLC - 8, Hopkins Symptom Checklist; SLEI, Stressful Life Events Inventory; SQA, Symptom Questionnaire Anxiety; SQD, Symptom Questionnaire Depression; SPI, Standardized Psychiatric Interview; SSI, Social Support Index; SSS, Social Support Survey; STAI, State-Trait Anxiety Inventory

* Correspondence to: Universidade do Minho Escola de Psicologia Campus de Gualtar 4710-057 Braga Portugal. Tel.: + 351253604656 ; fax: + 351253604224.

E-mail addresses: cdias@psi.uminho.pt (C.C. Dias), bbfi@psi.uminho.pt (B. Figueiredo).
} 


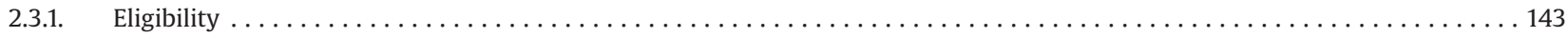

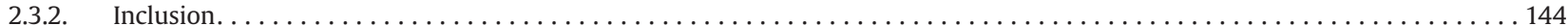

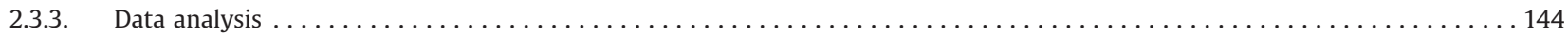

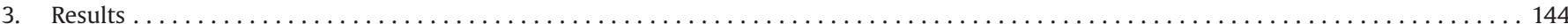

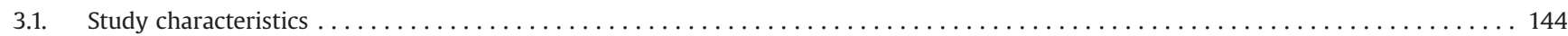

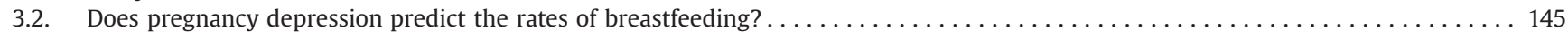

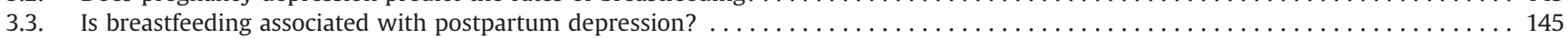

3.4. Does breastfeeding predict postpartum depression? or Does postpartum depression predict breastfeeding? ............. 147

3.5. Does pregnancy or postpartum depression predict breastfeeding and does breastfeeding predict postpartum depression? ....... 149

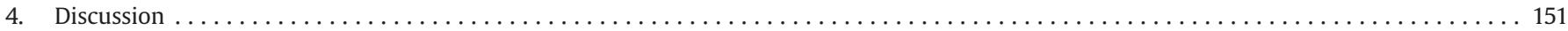

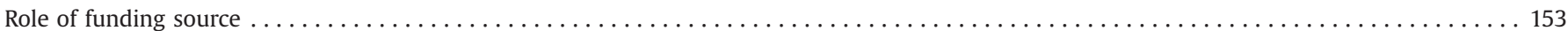

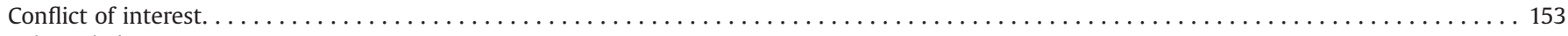

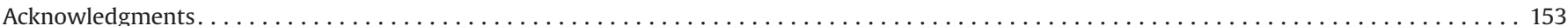

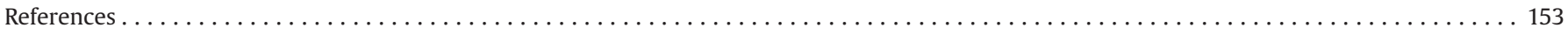

\section{Introduction}

Breastfeeding offers a wide range of benefits for both the child and the mother. The benefits for the infant include a diminished risk of infectious diseases and obesity and decreased blood pressure (Brion et al., 2011; Duijts et al., 2010; Horta et al., 2007). For the mother, breastfeeding confers a lower risk of ovarian and breast cancers and decreased blood pressure (Ebina and Kashiwakura, 2012; González-Jiménez et al., 2013; Jonas et al., 2008). Recognized as the optimal infant feeding method, the guidelines specified by the World Health Organization (WHO), the European Commission for Public Health (ECPH) and the American Academy of Pediatrics (AAP) recommend exclusive breastfeeding in the first 6 months postpartum (American Academy of Pediatrics, 2012; EU Project on Promotion of Breastfeeding in Europe, 2008; World Health Organization, 2007).

Although large variability across, most countries do not reach desirable rates of exclusive breastfeeding initiation and exclusive breastfeeding for 6 months (Cattaneo et al., 2005). Several studies have aimed to predict women at risk of no breastfeeding initiation or having an early cessation, given that this recommendation is not followed by most mothers (e.g., Bartick and Reinhold, 2010; Chalmers et al., 2009; Lee et al., 2013).

Pregnancy depression and postpartum depression appear to be possible significant contributors to this issue (Figueiredo et al., 2014; Hahn-Holbrook et al., 2013; Seimyr et al., 2004). It is widely known that pregnancy and postpartum depression have high incidence and that depressed women at pregnancy are usually depressed at the postpartum period (e.g., Figueiredo et al., 2007; Milgrom et al., 2008). Additionally, pregnancy and postpartum depression adverse effects have been consistently pointed out not only in breastfeeding, but also in mothers' behavior, health and psychological adjustment (e.g., Groer and Morgan, 2007), in infants' behavior and development (e.g., Figueiredo et al., 2010), and in the mother-infant interaction (e.g., Murray and Cooper, 1997).

To our knowledge, there are no published systematic reviews addressing the association among breastfeeding and pregnancy and postpartum depression. Given that pregnancy depression is the best predictor of postpartum depression (Figueiredo et al., 2007; Milgrom et al., 2008; Yonkers et al., 2001), it is important to simultaneously consider both pregnancy and postpartum depression in relation to breastfeeding in a review addressing the associations between these variables. This paper aimed to provide a systematic review of the literature on the association among breastfeeding and pregnancy and postpartum depression. Due to the associative nature of the majority of the published studies, it was not possible to perform a meta-analysis.

\section{Methods}

A total of 1673 relevant references were identified in an electronic search of three databases: MEDLINE, Web of Knowledge and PsycINFO. Duplicated references were removed and 771 articles remained. The titles and abstracts of the identified references were screened, and 707 non-relevant references were excluded. The full-text of the 65 remaining studies was then screened, and 17 studies met one or more exclusion criteria. At the final stage, 48 studies were included in the review. A flow diagram of the search selection for the included studies is presented in Fig. 1, and the procedures are described below.

\subsection{Data sources and search methodology (identification)}

An electronic search for empirical articles in MEDLINE, Web of Knowledge and PsycINFO from 1980 to December 2013 was performed according to the Preferred Reporting Items for Systematic Reviews and Meta-Analysis (PRISMA) statement (Moher et al., 2009). The search used the following keywords related to the review subject combined with standard MeSH terms: "breast feeding", "bottle feeding", "depression", "pregnancy", and "postpartum". The electronic search was independently performed by the first author (C.C.D.) and then replicated by the coauthor (B.F.).

\subsection{Study selection (screening)}

For the purpose of this review, only empirical studies that assessed the association among breastfeeding and pregnancy and/or postpartum depression were included. Different aspects of breastfeeding were considered-the intention, initiation, duration, confidence, self-efficacy, exclusivity or attitudes, as well as the different measures for pregnancy depression and postpartum depression. Studies were included regardless of the study design, the sample size or the measurement type. Only primary research was considered. Studies that met the following criteria were excluded: a) non-original research (review articles and meta-analysis) and b) studies focused on the effects of antidepressants on breastfeeding.

The included studies were assessed for quality based on the following criteria: 1) participants should be clearly defined as preor postpartum women; and 2) studies should identify the outcome measurements.

\subsection{Data extraction (eligibility and inclusion)}

\subsubsection{Eligibility}

In the first stage, the two authors (C.C.D. and B.F.) independently evaluated the titles and abstracts of all identified articles $(n=771)$ in order to assess potentially relevant references. 


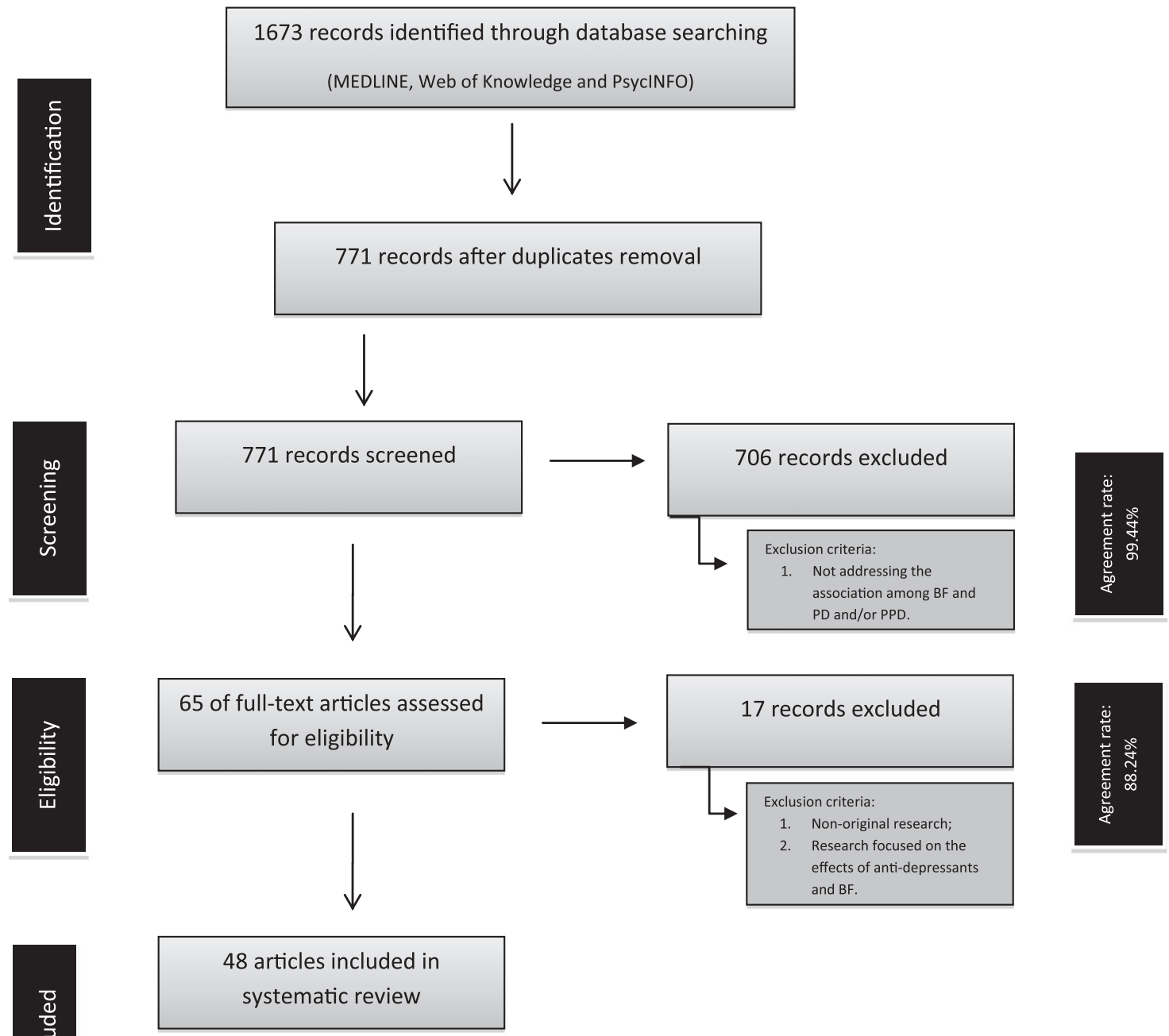

Fig. 1. Search strategy flow diagram.

Abstracts that did not address the association among breastfeeding and pregnancy depression and/or postpartum depression were automatically excluded at this stage $(n=706)$. The agreement rate between the two investigators at the first stage was $99.44 \%$.

In the second stage, the articles with abstracts that appeared relevant were selected for full-text evaluations $(n=65)$. Study selection was independently determined by the two authors based on the inclusion and exclusion criteria: non-original studies and studies focusing on the effects of antidepressants on breastfeeding were excluded $(n=17)$. The agreement rate between the two investigators was $88.24 \%$.

\subsubsection{Inclusion}

The two authors independently extracted data from the selected studies $(n=48)$ and fulfilled a standardized data extraction sheet. For each article, the extracted data included information about the authors, the publication year, the study's aim, the sample size and country, the measures and procedures, and the results.

The selected papers were organized according to the study aims and design. In these aspects, four main differences were found: prospective studies that analyzed if pregnancy depression predicts breastfeeding; associative studies analyzing the association between breastfeeding and postpartum depression; prospective studies on the prediction of breastfeeding and postpartum depression; and prospective studies taking into account both pregnancy and postpartum depression on the prediction of breastfeeding. Studies were then organized according to these four items: 1. Does pregnancy depression predict the rates of breastfeeding? 2. Is breastfeeding associated with postpartum depression? 3. Does breastfeeding predict postpartum depression? Does postpartum depression predict breastfeeding? and 4. Does pregnancy or postpartum depression predict breastfeeding and does breastfeeding predict postpartum depression? Studies were organized alphabetically by the first author.

At this stage, the agreement rate between the two investigators was $80.85 \%$. In all stages, in the case of a disagreement, a consensus was reached after discussion.

\subsubsection{Data analysis}

Quantitative results of each study regarding the association among breastfeeding and pregnancy and postpartum depression were retrieved. Both investigators performed registration of data.

\section{Results}

\subsection{Study characteristics}

The included studies were published between 1983 and 2013 in 19 different countries and evaluated a total of 71,245 participants. 
Table 1

Does pregnancy depression predict rates of breastfeeding (intention/initiation/duration)?

\begin{tabular}{|c|c|c|c|c|c|}
\hline $\begin{array}{l}\text { Authors/ } \\
\text { Title }\end{array}$ & Aims & Participants & Measures and Procedures & Results & Quality criteria \\
\hline $\begin{array}{l}\text { Fairlie } \\
\text { et al. } \\
\text { (2009) }\end{array}$ & $\begin{array}{l}\text { Impact of pregnancy } \\
\text { depression (PD) and high } \\
\text { pregnancy-related anxiety } \\
\text { on: } \\
\text { 1) Prenatal intention to } \\
\text { breastfeed } \\
\text { 2) Breastfeeding (BF) } \\
\text { initiation }\end{array}$ & $\begin{array}{l}1436 \\
\text { pregnant } \\
\text { women } \\
\text { (USA) }\end{array}$ & $\begin{array}{l}\text { Measures: brief interview; questionnaire on pregnancy- } \\
\text { related anxiety; questionnaire on history of depression; } \\
\text { questions about subject's intention to breastfeed; EPDS } \\
\text { ( } \geq 13 \text { ); information on BF initiation (postdelivery): BF } \\
\text { assessed by self-report measures and defined as a } \\
\text { dichotomous variable } \\
\text { Time-points: } 1 \text { st clinical prenatal appointment (usually in } \\
\text { the } 1 \text { st trimester gestation); } 26-28 \text { weeks gestation; post- } \\
\text { delivery }\end{array}$ & $\begin{array}{l}\mathrm{PD} \text { (EPDS } \geq 13 \text { at } 2 \text { nd } \\
\text { trimester gestation) } \\
\text { associated with } \downarrow \mathrm{BF} \\
\text { intention } \\
\mathrm{PD} \text { (EPDS } \geq 13 \text { at } 2 \text { nd } \\
\text { trimester gestation) did } \\
\text { not predict BF initiation }\end{array}$ & $\begin{array}{l}\text { Population with a BF } \\
\text { initiation rate } \\
\text { higher than the } \\
\text { national }\end{array}$ \\
\hline $\begin{array}{l}\text { Insaf } \\
\text { et al. } \\
(2011)\end{array}$ & $\begin{array}{l}\text { Association between } \\
\text { prenatal psychosocial risk } \\
\text { factors and BF intention }\end{array}$ & $\begin{array}{l}424 \text { pregnant } \\
\text { Hispanic } \\
\text { women } \\
\text { (USA) }\end{array}$ & $\begin{array}{l}\text { Measures: PSS; STAI; EPDS ( } \geq 13 \text { ); socio-demographic, } \\
\text { behavioral and acculturation information; BF intention } \\
\text { assessed by medical records and defined as a dichotomous } \\
\text { variable } \\
\text { Time-points: } 13.6 \text { weeks and } 25.7 \text { weeks gestation }\end{array}$ & $\begin{array}{l}\text { PD (EPDS } \geq 13 \text { at } 25.7 \\
\text { weeks) associated with } \downarrow \text { BF } \\
\text { intention }\end{array}$ & \\
\hline $\begin{array}{l}\text { Pippins } \\
\text { et al. } \\
(2006)\end{array}$ & $\begin{array}{l}\text { PD as a potential risk factor } \\
\text { for not initiating or } \\
\text { continuing } B F\end{array}$ & $\begin{array}{l}1448 \\
\text { pregnant } \\
\text { women } \\
\text { (USA) }\end{array}$ & $\begin{array}{l}\text { Measures: CES-D (>10); PSS; survey and medical record data; } \\
\text { BF assessed by self-report measures and defined as a } \\
\text { dichotomous variable } \\
\text { Time-points: } 15.6,26.1 \text {, and } 34 \text { weeks gestation and } 66.6 \\
\text { days postpartum }\end{array}$ & $\begin{array}{l}\text { PD }(C E S-D>10) \text { did not } \\
\text { predict BF initiation } \\
\text { PD }(C E S-D>10) \text { at } 2 \text { time } \\
\text { points (but not at } 1 \text { time- } \\
\text { point only) associated } \\
\text { with } \downarrow \text { BF duration } \\
(<1 \text { month) }\end{array}$ & $\begin{array}{l}\text { Early assessment of } \\
\text { BF duration }\end{array}$ \\
\hline
\end{tabular}

The majority of the studies were conducted in the United States $(n=15)$ and in the United Kingdom $(n=6)$. The remaining studies were published in Australia $(n=3)$, Brazil $(n=3)$, Canada $(n=3)$, Barbados $(n=2)$, Norway $(n=2)$, Pakistan $(n=2)$, Turkey $(n=2)$, China $(n=1)$, Congo $(n=1)$, Finland $(n=1)$, Iceland $(n=1)$, Italy $(n=1)$, Japan $(n=1)$, Mexico $(n=1)$, Portugal $(n=1)$, Sweden $(n=1)$, and the United Arab Emirates $(n=1)$.

Breastfeeding was assessed by self-report measures in 47 studies and by a medical records consultation in one study. Breastfeeding status was defined according to different criteria: Labbok and Krasovec criteria $(n=5)$; WHO recommendations $(n=1)$; as a dichotomous variable $(n=10)$; as a 3 or 4 group variable $(n=5)$; with the inclusion of solids $(n=2)$; exclusive breastfeeding status $(n=7)$; and not specified $(n=13)$. Breastfeeding duration was assessed at different postpartum time-points: before the 6 -month public recommendations $(n=26)$, and at 6 months $(n=12)$.

Thirty-nine studies assessed depression only through questionnaires, and nine studies used a clinical interview diagnosis for depression. Three studies assessed depression only during pregnancy, 35 studies measured it only at the postpartum period and nine studies assessed depression both during pregnancy and at the postpartum period. Pregnancy depressive symptoms were assessed during the three pregnancy trimesters $(n=2)$, during the 2 nd and 3 rd pregnancy trimesters $(n=2)$, during the 2 nd trimester $(n=4)$ or during the 3rd trimester $(n=3)$. Postpartum depressive symptoms were screened before the second month after childbirth $(n=15)$ and after the second month $(n=27)$.

\subsection{Does pregnancy depression predict the rates of breastfeeding?}

Three studies (see Table 1) were published on the prediction of breastfeeding by pregnancy depression without taking into account postpartum depression (Fairlie et al., 2009; Insaf et al., 2011; Pippins et al., 2006). Two studies highlighted breastfeeding intention and concluded that depression during pregnancy predicts shorter breastfeeding intention (Fairlie et al., 2009; Insaf et al., 2011). No association was found between pregnancy depression and breastfeeding initiation (Fairlie et al., 2009; Pippins et al., 2006). One study indicated that pregnancy depression predicts a shorter breastfeeding duration (Pippins et al., 2006) when depression occurred in more than one time point over pregnancy.

These studies showed that the association between pregnancy depression and breastfeeding intention and initiation is unclear, although pointed an association between pregnancy depression and a shorter breastfeeding duration.

\subsection{Is breastfeeding associated with postpartum depression?}

Eighteen studies (see Table 2) were found on the association among breastfeeding and postpartum depression that measured these variables at the same time-point (Astbury et al., 1994; Bick et al., 1998; Cooper et al., 1993; Dunn et al., 2006; Imbula et al., 2012; Feldens et al., 2011; Flores-Quijano et al., 2008; Hannah et al., 1992; Hatton et al., 2005; McCoy et al., 2006; McLearn et al., 2006; Misri et al., 1997; Papinczak and Turner, 2000; Taj and Sikander, 2003; Tamminen, 1988; Thome et al., 2006; Yonkers et al., 2001; Zubaran and Foresti, 2013).

A shorter breastfeeding duration was associated with higher rates of depressive symptoms and postpartum depression (Astbury et al., 1994; Bick et al., 1998; Cooper et al., 1993; Dunn et al., 2006; Imbula et al., 2012; Feldens et al., 2011; Flores-Quijano et al., 2008; Hannah et al., 1992; Hatton et al., 2005; McCoy et al., 2006; McLearn et al., 2006; Papinczak and Turner, 2000; Taj and Sikander, 2003; Thome et al., 2006; Yonkers et al., 2001; Zubaran and Foresti, 2013). Four studies referred specifically to the exclusive breastfeeding duration (Imbula et al., 2012; Flores-Quijano et al., 2008; Thome et al., 2006; Zubaran and Foresti, 2013) and concluded that postpartum depression and depressive symptomatology during the postpartum period were associated with early exclusive breastfeeding cessation. Three articles also concluded that the depressive symptoms were reported before the interruption of breastfeeding in the majority of the studied sample (Cooper et al., 1993; Misri et al., 1997; Taj and Sikander, 2003). Negative breastfeeding attitudes (Tamminen, 1988), breastfeeding difficulties (Tamminen, 1988), and a lower breastfeeding confidence (Flores-Quijano et al., 2008) have also been associated with more depressive symptoms and a higher incidence of postpartum depression.

These studies showed an unequivocal association between breastfeeding and postpartum depression. 
Table 2

Is breastfeeding associated with postpartum depression?

\begin{tabular}{|c|c|c|c|c|c|}
\hline $\begin{array}{l}\text { Authors/ } \\
\text { Title }\end{array}$ & Aims & Participants & Measures and Procedures & Results & $\begin{array}{l}\text { Quality } \\
\text { criteria }\end{array}$ \\
\hline $\begin{array}{l}\text { Astbury } \\
\text { et al. } \\
\text { (1994) }\end{array}$ & $\begin{array}{l}\text { Contribution of birth events, } \\
\text { satisfaction with care and social } \\
\text { differences to depression after } \\
\text { birth }\end{array}$ & $\begin{array}{l}790 \text { postpartum women } \\
\text { (Australia) }\end{array}$ & $\begin{array}{l}\text { Measures: EPDS ( } \geq 13 \text { ); BF status } \\
\text { not specified } \\
\text { Time-points: } 8-9 \text { months } \\
\text { postpartum }\end{array}$ & $\begin{array}{l}\downarrow \text { BF duration ( } 8-9 \text { months) } \\
\text { associated with postpartum } \\
\text { depression (PPD) (EPDS } \geq 13 \text { at } \\
8-9 \text { months postpartum) }\end{array}$ & $\begin{array}{l}\text { BF status not } \\
\text { specified }\end{array}$ \\
\hline $\begin{array}{l}\text { Bick et al. } \\
\text { (1998) }\end{array}$ & $\begin{array}{l}\text { Obstetric, maternal and social } \\
\text { factors associated with the } \\
\text { uptake and early cessation of BF } \\
\text { and women's reasons for } \\
\text { altering from BF to BoF }\end{array}$ & 906 postpartum women (UK) & $\begin{array}{l}\text { Measures: EPDS }(\geq 12) \text {; BF status } \\
\text { assessed by home-based interview } \\
\text { and defined as a dichotomous } \\
\text { variable } \\
\text { Time-points: a mean of } 11 \text { months } \\
\text { postpartum }\end{array}$ & $\begin{array}{l}\downarrow \text { BF duration ( }<3 \text { months) } \\
\text { associated with PPD (EPDS } \\
\geq 12 \text { in the first three months) }\end{array}$ & $\begin{array}{l}\text { Early } \\
\text { assessment } \\
\text { of BF } \\
\text { duration }\end{array}$ \\
\hline \multirow[t]{2}{*}{$\begin{array}{l}\text { Cooper } \\
\text { et al. } \\
(1993)\end{array}$} & \multirow[t]{2}{*}{$\begin{array}{l}\text { Association between } \\
\text { psychosocial factors and early } \\
\text { termination of BF }\end{array}$} & \multirow[t]{2}{*}{$\begin{array}{l}483 \text { postpartum women from } \\
2 \text { cohorts: Cambridge and Oxford } \\
\text { (UK) }\end{array}$} & $\begin{array}{l}\text { Measures: EPDS; PSE; SPI; BF status } \\
\text { not specified }\end{array}$ & $\begin{array}{l}\downarrow \text { BF duration (8 weeks } \\
\text { postpartum) associated with } \\
\text { PPD ( } 8 \text { weeks postpartum) }\end{array}$ & $\begin{array}{l}\text { Early } \\
\text { assessment } \\
\text { of BF } \\
\text { duration }\end{array}$ \\
\hline & & & Time-points: 8 weeks postpartum & $\begin{array}{l}\text { PPD preceded the cessation } \\
\text { of BF in a great majority of } \\
\text { women }\end{array}$ & $\begin{array}{l}\text { BF status not } \\
\text { specified }\end{array}$ \\
\hline $\begin{array}{l}\text { Dunn et al. } \\
\text { (2006) }\end{array}$ & $\begin{array}{l}\text { Vulnerability factors associated } \\
\text { with BF outcome at } 6 \text { weeks } \\
\text { postpartum after controlling for } \\
\text { age and education }\end{array}$ & $\begin{array}{l}526 \text { breastfeeding women } \\
\text { (Canada) }\end{array}$ & $\begin{array}{l}\text { Measures: telephone survey; EPDS } \\
\text { ( }>11) \text {; BF status defined as } 3 \text { groups: } \\
\text { exclusive BF, BF, and BoF } \\
\text { Time-point: } 6 \text { weeks postpartum }\end{array}$ & $\begin{array}{l}\downarrow \text { BF duration ( } 6 \text { weeks } \\
\text { postpartum) associated with } \\
\text { PPD (EPDS > } 11 \text { at } 6 \text { weeks } \\
\text { postpartum) }\end{array}$ & $\begin{array}{l}\text { Early } \\
\text { assessment } \\
\text { of BF } \\
\text { duration } \\
\text { Early } \\
\text { assessment } \\
\text { of PPD }\end{array}$ \\
\hline $\begin{array}{l}\text { Imbula } \\
\text { et al. } \\
\text { (2012) }\end{array}$ & $\begin{array}{l}\text { Frequency, risk factors and } \\
\text { clinical forms of PPD }\end{array}$ & 120 postpartum women (Congo) & $\begin{array}{l}\text { Measures: EPDS; SCID; GASD: } \\
\text { exclusive BF assessed by self-report } \\
\text { measures } \\
\text { Time-points: } 1-10 \text { months } \\
\text { postpartum }\end{array}$ & $\begin{array}{l}\downarrow \text { Exclusive BF duration (less } \\
\text { than } 6 \text { months) associated with } \\
\text { PPD (DSM IV criteria) (1-10 } \\
\text { months postpartum) }\end{array}$ & \\
\hline $\begin{array}{l}\text { Feldens } \\
\text { et al. } \\
(2011)\end{array}$ & Risk factors for discontinuing BF & 360 postpartum women (Brazil) & $\begin{array}{l}\text { Measures: socio-demographic and } \\
\text { BF interviews; BDI; BF status not } \\
\text { specified } \\
\text { Time-points: at birth, } 6 \text { and } 12 \\
\text { months postpartum }\end{array}$ & $\begin{array}{l}\downarrow \text { BF duration (12 months } \\
\text { postpartum) associated with } \\
\text { moderate (BDI } \geq 20 \text { ) to severe } \\
\text { (BDI } \geq 36 \text { ) depressive } \\
\text { symptoms ( } 12 \text { months } \\
\text { postpartum) }\end{array}$ & $\begin{array}{l}\text { BF status not } \\
\text { specified }\end{array}$ \\
\hline $\begin{array}{l}\text { Flores- } \\
\text { Quijano } \\
\text { et al. } \\
\text { (2008) }\end{array}$ & $\begin{array}{l}\text { Association between PPD, a } \\
\text { woman's confidence in her } \\
\text { ability to sustain lactation, BF } \\
\text { exclusiveness, and the sodium- } \\
\text { to-potassium (Na:K) ratio in milk }\end{array}$ & $\begin{array}{l}163 \text { postpartum BF women } \\
\text { (Mexico) }\end{array}$ & $\begin{array}{l}\text { Measures: EPDS ( } \geq 13 \text { ); } \\
\text { questionnaire on demographics and } \\
\text { infant feeding and hand-expressed } \\
\text { breast milk to assess BF exclusivity } \\
\text { Time-points: one assessment } \\
\text { between } 2 \text { and } 12 \text { weeks } \\
\text { postpartum }\end{array}$ & $\begin{array}{l}\downarrow \text { Exclusive BF duration ( } 2-12 \\
\text { weeks) associated with PPD } \\
\text { (EPDS } \geq 13 \text { at } 2-12 \text { weeks) } \\
\downarrow \text { BF confidence ( } 2-12 \text { weeks) } \\
\text { associated with PPD (EPDS } \\
\geq 13 \text { at } 2-12 \text { weeks) }\end{array}$ & $\begin{array}{l}\text { Early } \\
\text { assessment } \\
\text { of BF } \\
\text { duration } \\
\text { Early } \\
\text { assessment } \\
\text { of PPD }\end{array}$ \\
\hline $\begin{array}{l}\text { Hannah } \\
\text { et al. } \\
\text { (1992) }\end{array}$ & $\begin{array}{l}\text { Association between early post- } \\
\text { partum mood and PPD }\end{array}$ & 217 postpartum women (UK) & $\begin{array}{l}\text { Time-points: } 5 \text { days and } 6 \text { weeks } \\
\text { postpartum }\end{array}$ & $\begin{array}{l}\downarrow \text { BF duration ( } 1 \text { week } \\
\text { postpartum) associated with } \\
\uparrow \text { depressive symptoms ( } 1 \text { week } \\
\text { postpartum) } \\
\downarrow \text { BF duration ( }<6 \text { weeks } \\
\text { postpartum) associated with } \\
\text { PPD ( } 6 \text { weeks postpartum) }\end{array}$ & $\begin{array}{l}\text { Early } \\
\text { assessment } \\
\text { of BF } \\
\text { duration } \\
\text { Early } \\
\text { assessment } \\
\text { of PPD } \\
\text { BF status not } \\
\text { specified }\end{array}$ \\
\hline $\begin{array}{l}\text { Hatton } \\
\text { et al. } \\
(2005)\end{array}$ & $\begin{array}{l}\text { Association between depressive } \\
\text { symptoms and BF }\end{array}$ & 377 postpartum women (USA) & $\begin{array}{l}\text { Measures: EPDS ( } \geq 14 \text { ); BF status } \\
\text { not specified } \\
\text { Time-points: } 6 \text { and } 12 \text { weeks } \\
\text { postpartum }\end{array}$ & $\begin{array}{l}\downarrow \text { BF duration ( } 6 \text { weeks) } \\
\text { associated with PPD (EPDS } \\
\geq 14 \text { at } 6 \text { weeks) } \\
\text { BF duration ( }<12 \text { weeks) not } \\
\text { associated with PPD (EPDS } \\
\geq 14 \text { at } 12 \text { weeks) }\end{array}$ & $\begin{array}{l}\text { BF status not } \\
\text { specified }\end{array}$ \\
\hline $\begin{array}{l}\text { McCoy } \\
\text { et al. } \\
(2006)\end{array}$ & $\begin{array}{l}\text { Association between incidence } \\
\text { of PPD and age, BF status, } \\
\text { tobacco use, marital status, } \\
\text { history of depression, and } \\
\text { method of delivery }\end{array}$ & 209 postpartum women (USA) & $\begin{array}{l}\text { Measures: EPDS ( } \geq 13 \text { ), BF status } \\
\text { and socio-demographic information; } \\
\text { BF status not specified }\end{array}$ & $\begin{array}{l}\downarrow \text { BF duration ( } 4 \text { weeks } \\
\text { postpartum) associated with } \\
\text { PPD (EPDS } \geq 13 \text { at } 4 \text { weeks } \\
\text { postpartum) }\end{array}$ & $\begin{array}{l}\text { Early } \\
\text { assessment } \\
\text { of BF } \\
\text { duration } \\
\text { Early } \\
\text { assessment } \\
\text { of PPD } \\
\text { BF status not } \\
\text { specified }\end{array}$ \\
\hline $\begin{array}{l}\text { McLearn } \\
\text { et al. } \\
(2006)\end{array}$ & $\begin{array}{l}\text { Whether maternal depressive } \\
\text { symptoms are associated with } \\
\text { mothers' early parenting } \\
\text { practices }\end{array}$ & 5565 families (UK) & $\begin{array}{l}\text { Measures: Short questionnaire; } \\
\text { Interview about the presence of } \\
\text { depressive symptoms, early } \\
\text { parenting practices and additional } \\
\text { demographic characteristics; CES-D } \\
\text { ( } \geq 11 \text { ); BF status defined in } \\
4 \text { categories from } 2 \text { to } 4 \text { months: }\end{array}$ & $\begin{array}{l}\downarrow \text { BF duration (2-4 months } \\
\text { postpartum) associated with } \\
\text { PPD (CES-D } \geq 11 \text { at } 2-4 \\
\text { months postpartum) }\end{array}$ & $\begin{array}{l}\text { Early } \\
\text { assessment } \\
\text { of BF } \\
\text { duration }\end{array}$ \\
\hline
\end{tabular}


Table 2 (continued)

\begin{tabular}{|c|c|c|c|c|c|}
\hline $\begin{array}{l}\text { Authors/ } \\
\text { Title }\end{array}$ & Aims & Participants & Measures and Procedures & Results & $\begin{array}{l}\text { Quality } \\
\text { criteria }\end{array}$ \\
\hline $\begin{array}{l}\text { Misri et al. } \\
\text { (1997) }\end{array}$ & $\begin{array}{l}\text { Association between BF } \\
\text { cessation and the onset of PPD }\end{array}$ & $\begin{array}{l}51 \text { postpartum women meeting } \\
\text { DSMIV criteria for major } \\
\text { depression and who had stopped } \\
\text { BF (UK) }\end{array}$ & $\begin{array}{l}\text { "gave cereals"; "gave water", "gave } \\
\text { juice"; and "continued to BF" } \\
\text { Time-points: } 2-4 \text { months } \\
\text { postpartum } \\
\text { Measures: IFQ; CGI; BF exclusivity } \\
\text { assessed by self-report measures } \\
\text { Time-points: } 7.6 \text { months } \\
\text { postpartum and } 3 \text { months later }\end{array}$ & $\begin{array}{l}\text { PPD retrospectively reported } \\
\text { before the cessation of BF in } \\
83 \% \text { of the cases } \\
\text { Exclusive BF ( } 8 \text { weeks) } \\
\text { associated with less distress } \\
\text { surrounding BF process } \\
\text { Exclusive BF duration not } \\
\text { associated with PPD severity }\end{array}$ & $\begin{array}{l}\text { Early } \\
\text { assessment } \\
\text { of BF } \\
\text { duration }\end{array}$ \\
\hline $\begin{array}{l}\text { Papinczak } \\
\text { and } \\
\text { Turner } \\
\text { (2000) }\end{array}$ & $\begin{array}{l}\text { Association between certain } \\
\text { personal and social maternal } \\
\text { factors and the length of the } \\
\text { breastfeeding experience }\end{array}$ & $\begin{array}{l}159 \text { postpartum women } \\
\text { (Australia) }\end{array}$ & $\begin{array}{l}\text { Measures: qualitative and } \\
\text { quantitative data; BF status defined } \\
\text { as "exclusive" or "partial" } \\
\text { Time-points: at hospital discharge, } \\
3 \text { and } 6 \text { months postpartum }\end{array}$ & $\begin{array}{l}\text { associated with PPD severity } \\
\uparrow \mathrm{BF} \text { duration associated with } \\
\uparrow \mathrm{BF} \text { self-confidence } \\
\downarrow \mathrm{BF} \text { duration associated with } \\
\text { PPD }\end{array}$ & $\begin{array}{l}\text { Not } \\
\text { specification } \\
\text { of PPD } \\
\text { measurement }\end{array}$ \\
\hline $\begin{array}{l}\text { Taj and } \\
\text { Sikander } \\
\text { (2003) }\end{array}$ & $\begin{array}{l}\text { Effects of maternal depression } \\
\text { on BF behavior }\end{array}$ & $\begin{array}{l}100 \text { postpartum women } \\
\text { (Pakistan) }\end{array}$ & $\begin{array}{l}\text { Measures: HADS; Socio- } \\
\text { demographic information; BF status } \\
\text { not specified } \\
\text { Time-points: one assessment } \\
\text { between } 2 \text { months and } 2 \text { years } \\
\text { postpartum }\end{array}$ & $\begin{array}{l}\downarrow \text { BF duration ( } 2-12 \text { months } \\
\text { postpartum) associated with } \\
\uparrow \text { depressive symptoms ( } 2-12 \\
\text { months postpartum) } \\
36.8 \% \text { of non BF women } \\
\text { reported that their } \\
\text { depressive symptoms } \\
\text { preceded BF cessation }\end{array}$ & $\begin{array}{l}\text { BF status not } \\
\text { specified }\end{array}$ \\
\hline $\begin{array}{l}\text { Tamminen } \\
\text { (1988) }\end{array}$ & $\begin{array}{l}\text { Impact of mother's depression } \\
\text { on her BF and nursing attitudes }\end{array}$ & $\begin{array}{l}90 \text { women }-4 \text { groups: } 17 \text { in late- } \\
\text { pregnancy, } 10 \text { at } 1-10 \text { days } \\
\text { postpartum; } 30 \text { at } 2-4 \text { months } \\
\text { postpartum, and } 33 \text { at } 6-12 \\
\text { months postpartum (Finland) }\end{array}$ & $\begin{array}{l}\text { Measures: BDI; BF and childbearing } \\
\text { attitude scales. BF status not } \\
\text { specified } \\
\text { Time-points: at clinical } \\
\text { assessment and } 1-5 \text { weeks later }\end{array}$ & $\begin{array}{l}\text { BF difficulties (at the four } \\
\text { groups) associated with } \\
\uparrow \text { depressive symptoms } \\
\text { Negative BF attitudes (at the } \\
\text { four groups) associated with } \\
\uparrow \text { depressive symptoms }\end{array}$ & $\begin{array}{l}\text { BF status not } \\
\text { specified }\end{array}$ \\
\hline $\begin{array}{l}\text { Thome } \\
\text { et al. } \\
\text { (2006) }\end{array}$ & $\begin{array}{l}\text { Association between depressive } \\
\text { symptoms and parenting stress } \\
\text { and exclusive BF }\end{array}$ & $\begin{array}{l}734 \text { postpartum women } \\
\text { (Iceland) }\end{array}$ & $\begin{array}{l}\text { Measures: EPDS; PSI/SF; self-report } \\
\text { questionnaires on infant feeding } \\
\text { methods; demographic information; } \\
\text { BF status defined in } 4 \text { categories: } \\
\text { "Exclusive BF"; "Supplemented BF"; } \\
\text { "Bottle-feeding"; "Feeding of semi- } \\
\text { solids" } \\
\text { Time-points: } 2-3 \text { months } \\
\text { postpartum }\end{array}$ & $\begin{array}{l}\downarrow \text { Exclusive BF duration ( } 2-3 \\
\text { months postpartum) associated } \\
\text { with } \uparrow \text { depressive symptoms } \\
\text { ( } 2-3 \text { months postpartum) }\end{array}$ & $\begin{array}{l}\text { Early } \\
\text { assessment } \\
\text { of BF } \\
\text { duration }\end{array}$ \\
\hline $\begin{array}{l}\text { Yonkers } \\
\text { et al. } \\
\text { (2001) }\end{array}$ & $\begin{array}{l}\text { Risk factors for and rate of PPD } \\
\text { in a predominantly African } \\
\text { American and Hispanic clinic } \\
\text { population compared to } \\
\text { Caucasian women }\end{array}$ & $\begin{array}{l}802 \text { postpartum women from } \\
4 \text { inner-city community } \\
\text { maternal health clinics (USA) }\end{array}$ & $\begin{array}{l}\text { Measures: Demographic information } \\
\text { form; EPDS (>11); IDS; SCID; QLDS; } \\
\text { BF status not specified }\end{array}$ & $\begin{array}{l}\downarrow \text { BF duration ( } 3 \text { weeks) } \\
\text { associated with PPD (EPDS >11 } \\
\text { at } 3 \text { weeks postpartum) }\end{array}$ & $\begin{array}{l}\text { Early } \\
\text { assessment } \\
\text { of BF } \\
\text { duration } \\
\text { Early } \\
\text { assessment } \\
\text { of PPD }\end{array}$ \\
\hline & & & $\begin{array}{l}\text { Time-points: } 3 \text { weeks, } 4 \text { weeks and } \\
4-5 \text { weeks postpartum }\end{array}$ & & $\begin{array}{l}\text { BF status not } \\
\text { specified }\end{array}$ \\
\hline $\begin{array}{l}\text { Zubaran } \\
\text { and } \\
\text { Foresti } \\
(2013)\end{array}$ & $\begin{array}{l}\text { Association between BF self- } \\
\text { efficacy and PPD }\end{array}$ & $\begin{array}{l}89 \text { postpartum BF women } \\
\text { (Brazil) }\end{array}$ & $\begin{array}{l}\text { Measures: BSES-SF; EPDS }(\geq 13) \text {; } \\
\text { PDSS ( }>81 \text { ); SES; exclusive BF } \\
\text { assessed by self-report measures } \\
\text { Time-points: between } 2 \text { and } 12 \\
\text { weeks postpartum }\end{array}$ & $\begin{array}{l}\downarrow \text { Exclusive BF duration (2-12 } \\
\text { weeks) associated with } \\
\text { associated with } \uparrow \text { depressive } \\
\text { symptoms ( } 2-12 \text { weeks) } \\
\uparrow \text { Exclusive BF duration ( } 2-12 \\
\text { weeks) associated with } \\
\text { associated } \uparrow \text { BF self-efficacy } \\
(2-12 \text { weeks) }\end{array}$ & $\begin{array}{l}\text { Early } \\
\text { assessment } \\
\text { of BF } \\
\text { duration } \\
\text { Early } \\
\text { assessment } \\
\text { of PPD }\end{array}$ \\
\hline
\end{tabular}

\subsection{Does breastfeeding predict postpartum depression? or Does postpartum depression predict breastfeeding?}

Studies on the association between breastfeeding and postpartum depression (see Table 3), which measured depressive symptoms and breastfeeding at different time-points (Akman et al., 2008; Alder and Cox, 1983; Ali et al., 2009; Annagür et al., 2013; Chaudron et al., 2001; Dennis and McQueen, 2007; Gagliardi et al., 2012; Galler et al., 1999, 2006; Haga et al., 2012; Hasselmann et al., 2008; Henderson et al., 2003; Lau and Chan, 2007; McCarterSpaulding and Horowitz, 2007; Mezzacappa and Endicott, 2007; Nishioka et al., 2011; Taveras et al., 2003; Watkins et al., 2011), tried to determine the predictive value of breastfeeding and postpartum depression.

Regarding the predictive value of postpartum depression (assessed before breastfeeding duration assessment), nine studies showed that depressive symptoms or postpartum depression predicted a shorter breastfeeding duration (Akman et al., 2008; Dennis and McQueen, 2007; Gagliardi et al., 2012; Galler et al., 1999; Hasselmann et al., 2008; Henderson et al., 2003; McCarter-Spaulding and Horowitz, 2007; Nishioka et al., 2011; Taveras et al., 2003). Three studies reported specifically on depressive symptoms during the early postpartum period (Dennis and McQueen, 2007; Gagliardi et al., 2012; Hasselmann et al., 2008). One study found that depressive 
Table 3

Does breastfeeding predict further rates of postpartum depression? Does postpartum depression predict further patterns of breastfeeding?

\begin{tabular}{|c|c|c|c|c|c|}
\hline $\begin{array}{l}\text { Authors/ } \\
\text { Title }\end{array}$ & Aims & Participants & Measures and Procedures & Results & $\begin{array}{l}\text { Quality } \\
\text { criteria }\end{array}$ \\
\hline $\begin{array}{l}\text { Akman et al. } \\
\text { (2008) }\end{array}$ & $\begin{array}{l}\text { 1. Psychological adjustment } \\
\text { during early } \\
\text { postpartum months } \\
\text { 2. Effect of maternal depressive } \\
\text { symptoms, anxiety levels, } \\
\text { availability of social support } \\
\text { and maternal attachment on BF } \\
\text { discontinuation }\end{array}$ & $\begin{array}{l}60 \text { postpartum women } \\
\text { and their infants (Turkey) }\end{array}$ & $\begin{array}{l}\text { Measures: an initial assessment } \\
\text { interview; EPDS (at } 1 \text { month); STAI; } \\
\text { MSPSS; AAS; Exclusive BF assessed by } \\
\text { interview } \\
\text { Time-points: } 1 \text { week, } 1 \text { and } \\
4 \text { months postpartum }\end{array}$ & $\begin{array}{l}\downarrow \text { Exclusive BF duration ( } 4 \text { months) } \\
\text { associated with } \uparrow \text { depressive } \\
\text { symptoms ( } 1 \text { month postpartum) }\end{array}$ & $\begin{array}{l}\text { Early } \\
\text { assessment } \\
\text { of BF } \\
\text { duration } \\
\text { Early } \\
\text { assessment } \\
\text { of PPD }\end{array}$ \\
\hline $\begin{array}{l}\text { Alder and } \\
\text { Cox (1983) }\end{array}$ & Association between BF and PPD & $\begin{array}{l}89 \text { postpartum women } \\
\text { who took part on a } \\
\text { prospective study of PPD } \\
\text { (UK) }\end{array}$ & $\begin{array}{l}\text { Measures: EPDS; Exclusive BF defined } \\
\text { as total BF until } 12 \text { weeks } \\
\text { Time-points: } 18 \text { months }\end{array}$ & $\begin{array}{l}\text { Exclusive BF duration ( } 12 \text { weeks) } \\
\text { associated with higher incidence } \\
\text { of PPD ( } 18 \text { months) }\end{array}$ & \multirow[t]{2}{*}{$\begin{array}{l}\text { Early } \\
\text { assessment } \\
\text { of BF } \\
\text { duration }\end{array}$} \\
\hline $\begin{array}{l}\text { Ali et al. } \\
\text { (2009) }\end{array}$ & $\begin{array}{l}\text { Impact of postpartum anxiety and } \\
\text { depression on child growth and } \\
\text { development }\end{array}$ & $\begin{array}{l}420 \text { postpartum women } \\
\text { (Pakistan) }\end{array}$ & $\begin{array}{l}\text { Measures: Socio-demographic } \\
\text { questionnaire; home environment/ } \\
\text { family relationship questionnaire; } \\
\text { post-natal questionnaire; AKUADS } \\
\text { ( } \geq 19 \text { ) } \\
\text { Time-points: } 10 \text { days, } 1,2,6 \text { and } 12 \\
\text { months postpartum }\end{array}$ & $\begin{array}{l}\text { BF difficulties (birth) associated } \\
\text { with PPD (AKUADS } \geq 19 \text { at least } \\
\text { at one time-point) }\end{array}$ & \\
\hline $\begin{array}{l}\text { Annagür } \\
\text { et al. } \\
\text { (2013) }\end{array}$ & $\begin{array}{l}\text { Association between exclusive BF } \\
\text { and postpartum depressive } \\
\text { symptomatology }\end{array}$ & $\begin{array}{l}197 \text { postpartum women } \\
\text { (Turkey) }\end{array}$ & $\begin{array}{l}\text { Time-points: } 48 \mathrm{~h} \text { and } 6 \text { weeks } \\
\text { postpartum }\end{array}$ & $\begin{array}{l}\text { BF duration ( } 6 \text { weeks) not } \\
\text { associated with postpartum } \\
\text { depressive symptomatology ( } 48 \mathrm{~h} \\
\text { and } 6 \text { weeks) }\end{array}$ & $\begin{array}{l}\text { Early } \\
\text { assessment } \\
\text { of BF } \\
\text { duration } \\
\text { Early } \\
\text { assessment } \\
\text { of PPD }\end{array}$ \\
\hline $\begin{array}{l}\text { Chaudron } \\
\text { et al. } \\
\text { (2001) }\end{array}$ & $\begin{array}{l}\text { 1. Incidence of PPD } \\
\text { 2. Whether somatic complaints, } \\
\text { subsyndromal depressive } \\
\text { symptoms, or birth-related } \\
\text { concerns among non- } \\
\text { depressed women at } 1 \text { month } \\
\text { were predictive of PPD }\end{array}$ & $\begin{array}{l}465 \text { postpartum women } \\
\text { (USA) }\end{array}$ & $\begin{array}{l}\text { Measures: DIS; CES-D ( } \geq 16 \text { ); HRS; } \\
\text { measures specifically designed for } \\
\text { this study. BF status not specified } \\
\text { Time-points: } 1 \text { and } 4 \text { months } \\
\text { postpartum }\end{array}$ & $\begin{array}{l}\text { Bottle-feeding (BoF)/BF ( } 1 \text { month) } \\
\text { not associated with PPD (CES-D } \\
\geq 16 \text { at } 1 \text { and } 4 \text { months } \\
\text { postpartum) } \\
\text { BF worries ( } 1 \text { month) associated } \\
\text { with PPD (CES-D } \geq 16 \text { at } 1 \text { and } \\
4 \text { months postpartum) }\end{array}$ & $\begin{array}{l}\text { Early } \\
\text { assessment } \\
\text { of BF } \\
\text { duration } \\
\text { BF status } \\
\text { not } \\
\text { specified }\end{array}$ \\
\hline $\begin{array}{l}\text { Dennis and } \\
\text { McQueen } \\
\text { (2007) }\end{array}$ & $\begin{array}{l}\text { Association between diverse infant } \\
\text { feeding outcomes and PPD }\end{array}$ & $\begin{array}{l}594 \text { postpartum women } \\
\text { (Canada) }\end{array}$ & $\begin{array}{l}\text { Measures: EPDS ( }>12 \text { ); Infant } \\
\text { feeding method, maternal } \\
\text { satisfaction, infant feeding plans, BF } \\
\text { progress and BF self-efficacy; BF } \\
\text { status defined according to Labbok } \\
\text { and Krasovec criteria } \\
\text { Time-points: } 1,4 \text { and } 8 \text { weeks } \\
\text { postpartum }\end{array}$ & $\begin{array}{l}\text { BoF/ BF }(1 \text { week) not associated } \\
\text { with PPD (EPDS }>12 \text { at } 1,4 \text { or } \\
8 \text { weeks) } \\
\downarrow \text { BF duration ( }<4 \text { or } 8 \text { weeks) } \\
\text { associated with PPD (EPDS }>12 \\
\text { at } 1 \text { week) }\end{array}$ & $\begin{array}{l}\text { Early } \\
\text { assessment } \\
\text { of BF } \\
\text { duration } \\
\text { Early } \\
\text { assessment } \\
\text { of PPD }\end{array}$ \\
\hline \multirow[t]{2}{*}{$\begin{array}{l}\text { Gagliardi } \\
\text { et al. } \\
\text { (2012) }\end{array}$} & $\begin{array}{l}\text { Ability of the EPDS to predict later } \\
\text { BF problems }\end{array}$ & $\begin{array}{l}592 \text { postpartum women } \\
\text { (Italy) }\end{array}$ & $\begin{array}{l}\text { Measures: EPDS; BF status defined by } \\
\text { WHO }\end{array}$ & $\begin{array}{l}\downarrow \text { Exclusive BF duration ( } 3 \text { months) } \\
\text { associated with } \uparrow \text { depressive } \\
\text { symptoms ( } 2-3 \text { days postpartum) }\end{array}$ & $\begin{array}{l}\text { Early } \\
\text { assessment } \\
\text { of } \mathrm{BF} \\
\text { duration }\end{array}$ \\
\hline & & & $\begin{array}{l}\text { Time-points: } 2-3 \text { days and } 12-14 \\
\text { weeks postpartum }\end{array}$ & $\begin{array}{l}\text { The odds of BoF increased with } \\
\text { EPDS scores, even at low scores }\end{array}$ & $\begin{array}{l}\text { Early } \\
\text { assessment } \\
\text { of PPD }\end{array}$ \\
\hline $\begin{array}{l}\text { Galler et al. } \\
\text { (1999) }\end{array}$ & $\begin{array}{l}\text { Psychosocial variables affecting } \\
\text { early infant feeding practices in } \\
\text { Barbados }\end{array}$ & $\begin{array}{l}93 \text { postpartum women } \\
\text { and infants (Barbados) }\end{array}$ & $\begin{array}{l}\text { Measures: SDS; SAS; GAMS; } \\
\text { questionnaire to evaluate feeding } \\
\text { practices; BF status not specified } \\
\text { Time-points: } 7 \text { weeks, } 3 \text {, and } \\
6 \text { months postpartum }\end{array}$ & $\begin{array}{l}\text { BoF/BF ( } 7 \text { weeks, } 3 \text {, and } 6 \text { months } \\
\text { postpartum) predicted by PPD ( } 7 \\
\text { weeks) } \\
\text { BoF/BF ( } 7 \text { weeks) did not predict } \\
\text { PPD ( } 3 \text { and } 6 \text { months } \\
\text { postpartum) } \\
\text { PPD preceded BF cessation }\end{array}$ & $\begin{array}{l}\text { BF status } \\
\text { not } \\
\text { specified }\end{array}$ \\
\hline $\begin{array}{l}\text { Galler et al. } \\
\quad(2006)\end{array}$ & $\begin{array}{l}\text { Feeding attitudes at } 7 \text { weeks } \\
\text { postpartum and concurrent and } \\
\text { later feeding practices }\end{array}$ & $\begin{array}{l}226 \text { postpartum women } \\
\text { (Barbados) }\end{array}$ & $\begin{array}{l}\text { Measures: Questionnaire addressing } \\
\text { feeding practices; SDS; SAS; GAMS; } \\
\text { background variables; BF self-report } \\
\text { questionnaire; BF status unspecified } \\
\text { Time-points: birth, } 7 \text { weeks, } 3 \text { and } \\
6 \text { months postpartum }\end{array}$ & $\begin{array}{l}\text { Positive BF attitudes ( } 7 \text { weeks) } \\
\text { associated with } \downarrow \text { depressive } \\
\text { symptoms ( } 7 \text { weeks and } \\
6 \text { months) }\end{array}$ & $\begin{array}{l}\text { BF status } \\
\text { not } \\
\text { specified }\end{array}$ \\
\hline $\begin{array}{l}\text { Haga et al. } \\
\text { (2012) }\end{array}$ & $\begin{array}{l}\text { Multilevel model variations in } \\
\text { symptoms of PPD and use of } \\
\text { emotion regulation strategies, social } \\
\text { support, and BF self-efficacy }\end{array}$ & $\begin{array}{l}344 \text { postpartum women } \\
\text { (Norway) }\end{array}$ & $\begin{array}{l}\text { Measures: EPDS; CERQ; BSES; BSSS } \\
\text { Time-points: } 5-6 \text { weeks, } 3 \text { months } \\
\text { and } 6 \text { months postpartum }\end{array}$ & $\begin{array}{l}\text { BF self-efficacy ( } 5-6 \text { weeks, } 3 \text { and } \\
6 \text { months postpartum) predicted } \\
\downarrow \text { levels of depressive symptoms } \\
\text { (5-6 weeks, } 3 \text { and } 6 \text { months } \\
\text { postpartum) }\end{array}$ & \\
\hline $\begin{array}{l}\text { Hasselmann } \\
\text { et al. } \\
\text { (2008) }\end{array}$ & $\begin{array}{l}\text { PPD and risk of early interruption of } \\
\text { exclusive BF }\end{array}$ & 429 children (Brazil) & $\begin{array}{l}\text { Measures: Exclusive BF assed by } \\
\text { interview. EPDS ( } \geq 12) \text {; socio- } \\
\text { demographic information }\end{array}$ & $\begin{array}{l}\downarrow \text { Exclusive BF duration ( }<1 \text { month } \\
\text { and cumulative } 2 \text { months) } \\
\text { associated with PPD (EPDS } \geq 12 \\
\text { at early postpartum) }\end{array}$ & $\begin{array}{l}\text { Early } \\
\text { assessment } \\
\text { of } \mathrm{BF} \\
\text { duration }\end{array}$ \\
\hline
\end{tabular}


Table 3 (continued)

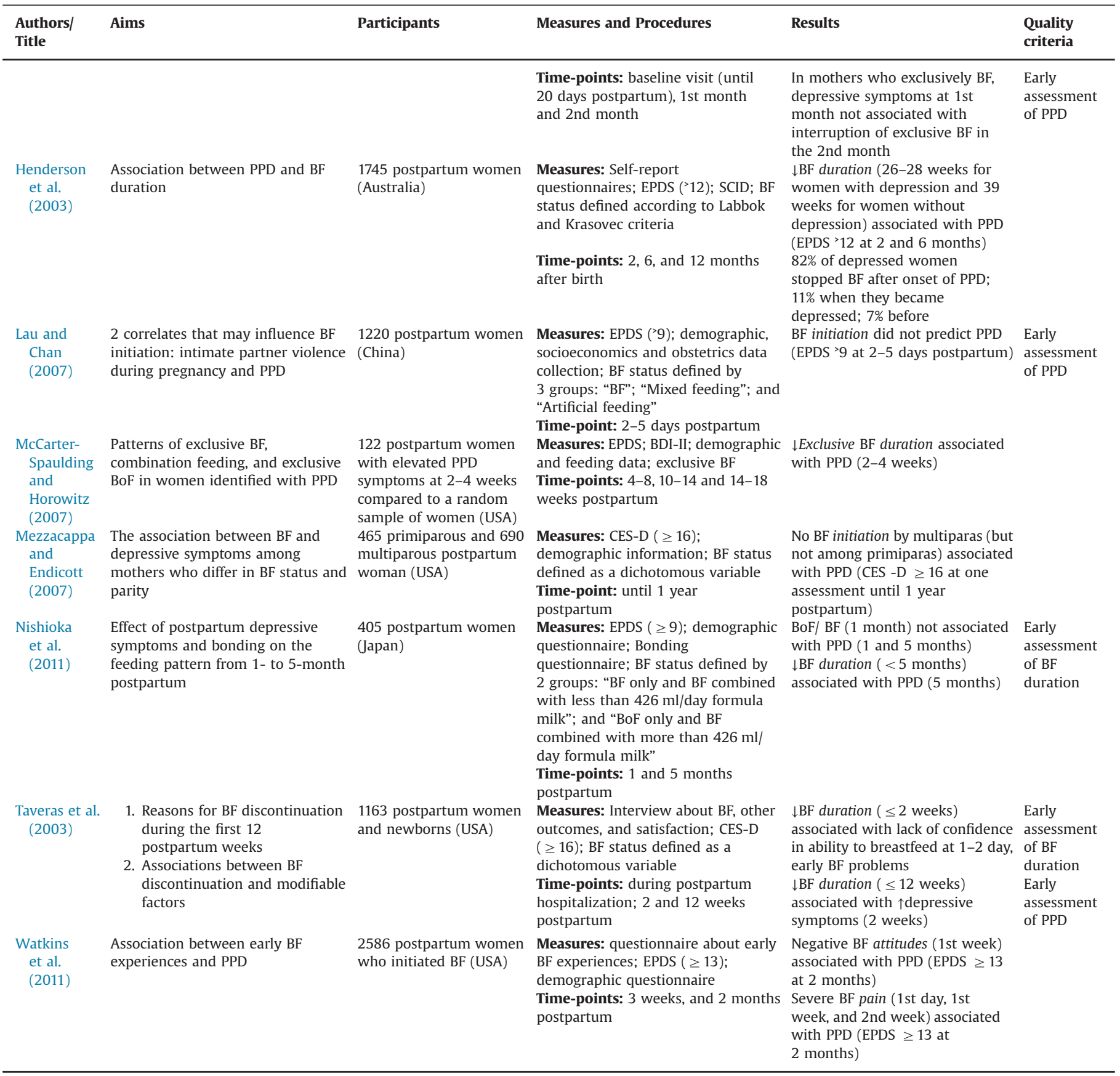

symptomatology at $48 \mathrm{~h}$ and 6 weeks did not predict further breastfeeding duration (Annagür et al., 2013).

Regarding the predictive value of breastfeeding (assessed before postpartum depression assessment), research remains equivocal. One study found that breastfeeding initiation was not associated with postpartum depressive symptoms (Lau and Chan, 2007), whereas another study concluded that breastfeeding initiation (only in multiparas) predicted lower postpartum depression (Mezzacappa and Endicott, 2007). Four studies found that breastfeeding or bottle-feeding status in the early postpartum period did not predict the development of further depressive symptoms (Chaudron et al., 2001; Dennis and McQueen, 2007; Galler et al., 1999; Nishioka et al., 2011). One study showed that exclusive breastfeeding duration predicted higher levels of postpartum depression (Alder and Cox, 1983). Breastfeeding worries (Chaudron et al.,
2001), negative breastfeeding attitudes (Galler et al., 2006; Watkins et al., 2011), breastfeeding difficulties (Ali et al., 2009), breastfeeding pain (Watkins et al., 2011), and a lower breastfeeding self-efficacy (Haga et al., 2012) were shown to predict higher levels of depressive symptoms or postpartum depression.

Prospective studies showed that depressive symptoms predict the early cessation of breastfeeding and that breastfeeding difficulties predict the development of depressive symptomatology.

\subsection{Does pregnancy or postpartum depression predict breastfeeding and does breastfeeding predict postpartum depression?}

Nine studies (see Table 4) highlighted the association between pregnancy depression and breastfeeding, also taking into account postpartum depression (Bogen et al., 2010; Chung et al., 2004; Field 
Table 4

Does pregnancy depression predict rates of breastfeeding?/Is breastfeeding associated with postpartum depression?

\begin{tabular}{|c|c|}
\hline $\begin{array}{l}\text { Authors/ } \\
\text { Title }\end{array}$ & Aims \\
\hline $\begin{array}{l}\text { Bogen } \\
\text { et al. } \\
(2010)\end{array}$ & $\begin{array}{l}\text { Association between: } \\
\text { 1. Major depressive disorder and } \\
\text { depressive symptom severity } \\
\text { during pregnancy and BF intention; } \\
\text { 2. Major depressive disorder and } \\
\text { depressive symptom severity } \\
\text { during pregnancy and BF initiation } \\
\text { and status; } \\
\text { 3. Serotonin reuptake inhibitor use } \\
\text { and BF intention, initiation } \\
\text { and status }\end{array}$ \\
\hline $\begin{array}{l}\text { Chung } \\
\text { et al. } \\
\text { (2004) }\end{array}$ & $\begin{array}{l}\text { Association between maternal } \\
\text { depressive symptoms and the use of } \\
\text { infant health services, parenting } \\
\text { practices, and injury- prevention } \\
\text { measures }\end{array}$ \\
\hline $\begin{array}{c}\text { Field et al. } \\
\text { (2002) }\end{array}$ & $\begin{array}{l}\text { 1. Incidence of BF in a sample of } \\
\text { pregnant depressed woman } \\
\text { 2. Duration of BF, mother's self- } \\
\text { reported confidence in BF, and } \\
\text { perception of the infant's } \\
\text { temperament. }\end{array}$ \\
\hline
\end{tabular}

Figueiredo

et al.

(2014)
1. Effects of PD and PPD on exclusive 145 BF initiation, early cessation pregnant and length 2. Effects of exclusive BF initiation and (Portugal) early cessation on PPD.

$\begin{array}{ll}\text { Participants Measures and Procedures } & \text { Results }\end{array}$

238 Measures: Feeding intention survey;

pregnant

women

(USA)

774 pregnant women (USA)

40 pregnant women (USA)
Feeding practices survey; SCID; HDRS;
LIFE; BF status defined by 4 groups:

"Breast"; "Breast and formula";

"Formula"; and "Uncertain"

Time-points: 20, 30, and 30 weeks

gestation, 2 and 12 weeks postpartum
PD and depressive symptom severity not Early BF associated with $\mathrm{BF}$ intention duration PD and PPD (at 2 or 12 weeks assessment postpartum) not associated with $\mathrm{BF}$ initiation or BF duration at 2 or 12 weeks
Measures: structured surveys; CES-D $(\geq 16)$; BF status not specified Time-points: pregnancy, 3-4 months and 9-12 months postpartum

Measures: CES-D ('16) SCID; phone with $\uparrow$ scores of depressive symptoms at self-perceptions about $\mathrm{BF}$ confidence and 8 months postpartum infant behavior during BF; MABS; BF status defined according to Labbok and Krasovec criteria

Time-points: 21 weeks gestation and 8 months postpartum

Measures: Socio-demographic

questionnaire; EPDS; BF status defined according to Labbok and Krasovec criteria

Time-points: 1st, 2nd and 3rd pregnancy trimester, at childbirth and 3,6 , and 12 moths postpartum

PD (CES-D >16 at 21 weeks) associated with $\downarrow$ BF duration at 8 months postpartum

$\uparrow$ Depression scores (1st trimester) predicted $\uparrow$ exclusive BF duration (3 months postpartum)

$\uparrow$ Depression scores (3rd trimester) associated with $\downarrow$ BF duration BF initiation associated with a decrease on depression scores (from 1st pregnancy trimester to 3-months

\section{Hahn- \\ Hol- \\ brook \\ et al. \\ (2013)}

1. Effects of early BF behaviors on 205 later depressive symptomatology in pregnant mothers

2. Effects of PD on later BF behaviors

3. Which variables could account for the association between BF and depression

$\begin{aligned} & \text { Hamdan } \\ & \text { and }\end{aligned}$
Tamin
$(2011)$

1. Period and point prevalence of maternal depressive mood before and after childbirth

2. Association to the parent's psychosocial conditions and experiences of parenthood
, women SSS; BF status defined according to (USA) Labbok and Krasovec criteria Time-points: 15, 20, 25, 31 and 37 weeks of gestation and at $3,6,12$, and 24 months postpartum

137
pregnant
women
(United
Arab
Emirates)

780 postpartum women (Canada)

434 pregnant women (Sweden)
Measures: Socio-demographic questionnaire; BDI-II; BAI; SLEI; Selfreported religiosity; EPDS; MINI; postnatal questionnaire; BF status defined as dichotomous variable

Time-points: 2nd and 3rd trimester gestation; 2 and 4 months postpartum Measures: SQD ( > 8.30); SQA ( > 11.58); SSI ( $<$ 33rd percentile of scores); RSES ( $<33$ rd percentile of scores); EPDS ( $\geq 13$ ); socio-demographic information; BF status defined as a dichotomous variable

Time-points: study intake, 32-36 weeks gestation, 8 weeks, and 3 years postpartum

Measures: EPDS ( $\geq 10$ ); EMQ/EFQ; questionnaire about background and psychosocial conditions in the life situation; BF status not specified Time-points: pregnancy ( $>30$ weeks gestation), 2 and 12 months postpartum
PD (CES-D $\geq 16$ at pregnancy) and PPD Early BF (CES-D $\geq 16$ at 3-4 and 9-12 months) not duration associated with BF duration ( $\geq 1$ month) assessment BF status not specified postpartum), while no significant differences in depression scores for women who did not initiate BF $\uparrow$ Depression symptoms scores (1st trimester and childbirth) associated with no $\mathrm{BF}$ initiation or $\downarrow$ exclusive $\mathrm{BF}$ duration

$\uparrow$ Depression scores (3rd trimester) were the best predictors of lower BF duration PD (CES-D $\geq 4$ at any pregnancy timepoint) associated with $\downarrow$ BF duration (3 months), but not between 3 and 12 months

$\uparrow \mathrm{BF}$ duration (3 or more months) associated with $\downarrow$ depression symptoms scores (24 months postpartum) Depressive symptoms (3 months) not associated with later BF duration

$\mathrm{BF}$ initiation and $\uparrow \mathrm{BF}$ duration $(<2$ months) associated with $\downarrow$ depressive

Early BF duration symptoms scores at 2 months postpartum assessment

PD (SQD > 8.30 at pregnancy) associated with $\downarrow$ BF duration ( $<6$ months)

PPD (EPDS $\geq 13$ ) not associated with $\downarrow$ BF duration ( $<6$ months)

PD (EPDS $\geq 10$ at 30 weeks) associated with $\downarrow$ BF initiation and $\uparrow \mathrm{BF}$ difficulties;

Early BF duration assessment

PPD (EPDS $\geq 10$ at 2 months postpartum) BF status associated with $\downarrow$ BF initiation, $\uparrow \mathrm{BF}$ not difficulties, and $\downarrow$ BF duration ( $<5$ months) specified
Early BF duration assessment 
Table 4 (continued)

\begin{tabular}{|c|c|c|c|c|c|}
\hline $\begin{array}{l}\text { Authors/ } \\
\text { Title }\end{array}$ & Aims & Participants & Measures and Procedures & Results & $\begin{array}{l}\text { Quality } \\
\text { criteria }\end{array}$ \\
\hline $\begin{array}{l}\text { Ystrom } \\
\quad(2012)\end{array}$ & $\begin{array}{l}\text { 1. Association between BF cessation } \\
\text { and an increase in symptoms of } \\
\text { anxiety and depression from } \\
\text { pregnancy to } 6 \text { months postpartum } \\
\text { 2. The disproportionately high } \\
\text { proposed symptom increase after } \\
\text { BF cessation for women already } \\
\text { suffering from high levels of } \\
\text { prepartum anxiety and depression }\end{array}$ & $\begin{array}{l}42,225 \\
\text { pregnant } \\
\text { women } \\
\text { (Norway) }\end{array}$ & $\begin{array}{l}\text { Measures: ultrasound examination; self- } \\
\text { report questionnaires; SLC - 8; BF status } \\
\text { defined by } 3 \text { groups: "Predominant BF"; } \\
\text { "Mixed BF"; "Bottle-feeding" } \\
\text { Time-points: } 30 \text { weeks gestation and } \\
6 \text { months postpartum }\end{array}$ & $\begin{array}{l}\text { Depressive symptoms ( } 30 \text { weeks } \\
\text { gestation) associated with } \downarrow \text { BF duration } \\
\text { ( }<6 \text { months) } \\
\downarrow \text { BF duration ( }<6 \text { months) predicted } \\
\uparrow \text { depressive symptoms ( } 6 \text { months } \\
\text { postpartum) } \\
\text { Anxiety and depressive symptoms } \\
\text { during pregnancy interacted with the } \\
\text { relation between BF cessation and } \\
\text { postpartum anxiety and depression, in } \\
\text { terms that pregnancy anxiety and } \\
\text { depression baseline levels are increased } \\
\text { at } 6 \text { months postpartum by BF cessation }\end{array}$ & \\
\hline
\end{tabular}

et al., 2002; Figueiredo et al., 2014; Hamdan and Tamin, 2011; HahnHolbrook et al., 2013; Kehler et al., 2009; Seimyr et al., 2004; Ystrom, 2012). Only one study examined the association between pregnancy depression and breastfeeding intention and found no association (Bogen et al., 2010). Research on the association between pregnancy depression and breastfeeding initiation remains equivocal, with one study showing no association between the variables (Bogen et al., 2010) and two studies suggesting that depressive symptomatology during pregnancy and pregnancy depression predicted shorter breastfeeding initiation (Figueiredo et al., 2014; Seimyr et al., 2004). The association between pregnancy depression and breastfeeding duration was analyzed in seven studies. Two studies found no association between these variables (Bogen et al., 2010; Chung et al., 2004), and five studies concluded that depressive symptomatology during pregnancy or pregnancy depression predicted shorter breastfeeding duration (Field et al., 2002; Figueiredo et al., 2014; Hahn-Holbrook et al., 2013; Kehler et al., 2009; Ystrom, 2012). One of the articles specifically measured exclusive breastfeeding duration (Figueiredo et al., 2014). Pregnancy depression also predicted more breastfeeding difficulties (Seimyr et al., 2004).

Seven studies aimed to investigate the association between breastfeeding and postpartum depression, also taking into account pregnancy depression (Bogen et al., 2010; Chung et al., 2004; Figueiredo et al., 2014; Hamdan and Tamin, 2011; Hahn-Holbrook et al., 2013; Seimyr et al., 2004; Ystrom, 2012). Two studies showed that no breastfeeding initiation predicted postpartum depression (Hamdan and Tamin, 2011; Seimyr et al., 2004), while another study found no association between these variables (Bogen et al., 2010). A shorter breastfeeding duration was associated with postpartum depression in some studies (Figueiredo et al., 2014; Hamdan and Tamin, 2011; Seimyr et al., 2004; Ystrom, 2012), but not in others (Bogen et al., 2010; Hahn-Holbrook et al., 2013; Kehler et al., 2009). One study showed that postpartum depression predicted a shorter breastfeeding duration (Seimyr et al., 2004). Another study found that shorter breastfeeding duration did not predict postpartum depression (Chung et al., 2004). Breastfeeding difficulties were also associated with postpartum depression (Seimyr et al., 2004). Two studies showed that the association between pregnancy depression and postpartum depression is mediated by breastfeeding (Figueiredo et al., 2014; Ystrom, 2012). One study (Figueiredo et al., 2014) reported that breastfeeding initiation decreases depression scores from pregnancy to postpartum, and the other study (Ystrom, 2012) showed that breastfeeding cessation increases depressive symptoms from pregnancy to postpartum.

These studies found that pregnancy depression predicted shorter breastfeeding duration and that breastfeeding initiation and longer duration predicted a decrease on depressive symptomatology during the postpartum.

\section{Discussion}

This systematic review provides an overview of the current knowledge on the associations among breastfeeding and pregnancy or postpartum depression. It includes studies from several countries published over a 30-year period. However, the four selected keywords may have led to the exclusion of relevant references. The use of broader keywords such as "maternal mental health", "psychological wellbeing", or "maternal depression" could have led to the identification of more records. Despite methodological differences between the included studies (e.g., differences in the studies' designs, and in breastfeeding and pregnancy and postpartum depression assessment measures), an association between breastfeeding and depression during both the prenatal and postpartum periods is suggested.

Does pregnancy depression predict the rates of breastfeeding? Research on the prediction of breastfeeding intention, initiation, and duration by pregnancy depression remains scarce or presents methodological differences, providing some unclear results. The only clear prediction this review found was with regards to breastfeeding duration (Pippins et al., 2006), particularly in studies that consider both pregnancy and postpartum depression as reported later.

Is breastfeeding associated with postpartum depression? The association between breastfeeding and postpartum depression seems to be clear. Early exclusive and non-exclusive breastfeeding cessation is associated with the presence of postpartum depression in all studies published in the last 30 years (Astbury et al., 1994; Bick et al., 1998; Cooper et al., 1993; Dunn et al., 2006; Imbula et al., 2012; Feldens et al., 2011; Flores-Quijano et al., 2008; Hamdan and Tamin, 2011; Hannah et al., 1992; Hatton et al., 2005; McCoy et al., 2006; McLearn et al., 2006; Papinczak and Turner, 2000; Seimyr et al., 2004; Taj and Sikander, 2003; Thome et al., 2006; Yonkers et al., 2001; Ystrom, 2012; Zubaran and Foresti, 2013).

Does breastfeeding predict postpartum depression? or Does postpartum depression predict breastfeeding? In several studies, postpartum depressive symptoms were shown to predict early breastfeeding cessation (Akman et al., 2008; Dennis and McQueen, 2007; Gagliardi et al., 2012; Galler et al., 1999; Hasselmann et al., 2008; Henderson et al., 2003; McCarter-Spaulding and Horowitz, 2007; Nishioka et al., 2011; Taveras et al., 2003), with the exception of one study (Annagür et al., 2013). These studies offer trends suggesting that depressive symptoms precede and lead to the early cessation of breastfeeding.

Negative breastfeeding experiences were also reported to precede the onset of depressive symptomatology. In particular, breastfeeding worries (Chaudron et al., 2001), difficulties (Ali et al., 2009), negative attitudes (Galler et al., 2006; Watkins et al., 2011), 
pain (Watkins et al., 2011), and a low self-efficacy (Haga et al., 2012) preceded the emergence of depressive symptoms.

Two studies focused on the prediction of depressive symptoms by breastfeeding initiation and provided contradictory results (Lau and Chan, 2007; Mezzacappa and Endicott, 2007). One study found no prediction (Lau and Chan, 2007), while the other study showed that breastfeeding initiation by multiparas predicted lower levels of depressive symptoms during the postpartum period (Mezzacappa and Endicott, 2007). Moreover, later breastfeeding or bootle-feeding status was not shown to predict postpartum depression in other studies as well (Chaudron et al., 2001; Dennis and McQueen, 2007; Galler et al., 1999; Nishioka et al., 2011). An imprecise breastfeeding definition and timing of measurement (without indication regarding breastfeeding initiation) are important limitations of these studies. More research is warranted on the association between breastfeeding initiation and the further onset of depressive symptoms.

Does pregnancy or postpartum depression predict breastfeeding and does breastfeeding predict postpartum depression? Pregnancy and postpartum depression are both associated with a shorter breastfeeding duration in most of the selected studies (Field et al., 2002; Figueiredo et al., 2014; Hamdan and Tamin, 2011; Hahn-Holbrook et al., 2013; Kehler et al., 2009; Seimyr et al., 2004; Ystrom, 2012). The only two studies (Bogen et al., 2010; Chung et al., 2004) that found no association among breastfeeding and pre- and postnatal depression measured breastfeeding duration at 1 and 2 months postpartum and did not indicate if the 6 month usual recommendation was fulfilled by depressed women. Moreover, despite no significant association among breastfeeding and pre- and postnatal depression, Chung et al. (2004) showed an overall trend towards a decrease in breastfeeding with the increase in postpartum depressive symptoms.

Some recent studies showed that pregnancy depression is a stronger predictor than postpartum depression for a shorter breastfeeding duration (e.g., Figueiredo et al., 2014; Kehler et al., 2009; Hahn-Holbrook et al., 2013), with pregnant depressed women at higher risk for a shorter breastfeeding duration. These studies claim the need to identify depression early during pregnancy to detect women at higher risk for a shorter breastfeeding duration and to identify the mechanisms underlying breastfeeding behavior in these women. Moreover, recent evidence also indicates that when both antenatal and postnatal depression are taken into account, breastfeeding may act as a moderator factor, decreasing or increasing depressive symptoms from pre- to postpartum (Figueiredo et al., 2014; Hahn-Holbrook et al., 2013; Ystrom, 2012).

In conclusion, trends were found regarding symptoms of depression both during pregnancy and the postpartum period having a negative impact on breastfeeding. Women with depressive symptomatology both before and after childbirth are at higher risk of breastfeeding discontinuation. Study design revealed some important patterns between studies. Prospective studies analyzing the prediction of breastfeeding by pregnancy depression showed that prenatal depression predicts shorter breastfeeding duration. However, they did not explain postpartum depressive symptoms' role on this prediction, as they did not assess for postpartum depressive symptoms and/or postpartum depression. Associative studies found a correlation between breastfeeding duration and postpartum depression, although not explaining the direction of this association. Prospective studies analyzing the impact of postpartum depression on breastfeeding and the impact of breastfeeding on postpartum depression showed that postpartum depression and postpartum depressive symptoms predict lower breastfeeding duration. Moreover, these studies revealed that experiencing breastfeeding problems (e.g., breastfeeding difficulties, pain, worries, low self-efficacy, and negative attitudes) could also expose women to a higher risk of developing postpartum depression. However, as they did not assess for pregnancy depressive symptoms and/or pregnancy depression, these studies did not explain the effect of prenatal depression on breastfeeding duration and/or breastfeeding problems. Prospective research taking into account both pregnancy and postpartum depression pointed the role of depressive symptoms during pregnancy on breastfeeding duration and the role of breastfeeding duration on depressive symptomatology maintenance through the postpartum period. These studies give important clues on the direction of the association among breastfeeding and pregnancy and postpartum depression, as they brought initial evidence that in women who were depressed during pregnancy, breastfeeding could act as an important factor for decreasing depressive symptoms after birth. However, these findings still require further research, and additional empirical evidence is warranted, specifically prospective studies taking into account both the pregnancy and postpartum periods. Pregnancy depression has also been referred as the strongest risk factor for postpartum depression in the literature. There is evidence that pregnancy depression plays an important role in the association between breastfeeding and postpartum depression, and some studies have shown that pregnancy depression is a strong predictor for shorter breastfeeding duration, even when postpartum depression was considered.

In general, research showed an association among breastfeeding and pregnancy and postpartum depression. However, there are still some equivocal findings that remain to be explained. Methodological differences across the studies may help to clarify circumstances associated with non-replicate results.

Regarding participants, some studies used homogeneous samples or samples with a breastfeeding initiation rate higher than the national average, thereby compromising the generalization of the results.

With regards to the variables' measurement, both breastfeeding and pregnancy and postpartum depression were assessed using a wide range of measures and at different time points during pregnancy and the postpartum period. Breastfeeding, for example, was assessed with very different criteria: some studies assessed breastfeeding according to Labbok and Krasovec or WHO standards, whereas other studies assessed breastfeeding as a dichotomous variable (e.g., "yes/no"), which lead to an imprecise definition. Studies varied in terms of the time point assessment as well: some studies assessed breastfeeding at the 6-month usual recommendation and other studies assessed breastfeeding earlier. Studies also used a wide range of self-report measures and only $19 \%$ a clinical interview diagnosis for depression. In addition, depressive symptoms were assessed at different time points after childbirth, and not all studies addressed postpartum depression. The postpartum period is a critical period for the onset of depressive symptoms, and postpartum depression has been reported to begin between the second and the third months postpartum (Kumar and Robson, 1984; O'Hara, 1997; Pitt, 1968). When measuring depressive symptoms earlier than the first month postpartum, studies are addressing Postpartum Blues (Kennerley and Gath, 1989; Yalom et al., 1968), which is not a depressive disorder.

Some studies screened for the mother's anxiety (e.g., Ali et al., 2009; Fairlie et al., 2009; Insaf et al., 2011; Kehler et al., 2009; Papinczak and Turner, 2000; Ystrom, 2012). These studies found an association between the mother's anxiety and increased breastfeeding difficulties, as well as shorter breastfeeding intention and duration. Research has shown that depression and anxiety symptoms are often comorbid (Austin et al., 2010; Figueiredo and Conde, 2011). Moreover, recent literature suggested an association between maternal anxiety and a shorter breastfeeding duration (Adedinsewo et al., 2014; Paul et al., 2013).

Methodological differences between the selected studies may help to clarify some important differences between them. For example, the only study that found a positive association between 
breastfeeding duration and postpartum depression used a small sample. On its turn, when we look to studies that show no association between PD or postpartum depression and breastfeeding duration or breastfeeding/bottle-feeding status (Annagür et al., 2013; Bogen et al., 2010; Chaudron et al., 2001; Chung et al., 2004; Dennis and McQueen, 2007; Galler et al., 1999; Hatton et al., 2005; Kehler et al., 2009; Nishioka et al., 2011) we can see that the majority assessed breastfeeding duration early in the postpartum (1 week to 6 weeks) not addressing breastfeeding maintenance but breastfeeding initiation. Perhaps the mechanisms underlying breastfeeding initiation could be different than those underlying breastfeeding duration. Moreover, almost all of these studies have an imprecise definition of breastfeeding or defined it as a dichotomous variable, and none of them assessed breastfeeding exclusivity, giving no indications if pregnancy and/or postpartum depression predicts early cessation of exclusive breastfeeding.

On the other hand, differences on depression assessment may reveal different results. With the exception of one (Bogen et al., 2010), all studies that used a clinical diagnosis interview for depression found an association among pregnancy or postpartum depression and breastfeeding duration.

According to these methodological issues, to use standard criteria and measurements for both breastfeeding and postpartum depression is main concern for future research. To specifically address exclusive breastfeeding for at least 6 months according to the standards and to use a clinical interview diagnosis tool (and not only self-report measures) between the second and third months postpartum to effectively diagnose postpartum depression are specific recommendations. Researchers should also use representative samples in order to draw unbiased conclusions that can be generalized. Special attention for the effect of parity and anxiety in the association among breastfeeding and pregnancy and postpartum depression should also be given.

Breastfeeding carries a wide range of benefits for both the mother and the child, and exclusive breastfeeding is recommended for the first 6 months of an infant's life. The identification of risk factors for early breastfeeding cessation is a health priority. Screening for depression during pregnancy could be a useful tool to identify women at risk for both a shorter breastfeeding duration and postpartum depression. Experiencing breastfeeding problems can also put women at risk for depressive symptoms in the postpartum period. This review supports the need to identify and help women with depressive symptoms during pregnancy or facing problems with breastfeeding at early postpartum in order to enhance breastfeeding and promote postpartum psychological adjustment.

\section{Role of funding source}

This research was supported by FEDER Funds through the ProgramaOperacionalFactores de Competitividade - COMPETE and by National Funds through FCT Fundaçãopara a Ciência e a Tecnologia under the project: PTDC/SAU/SAP/116738/2010.

The sponsors had no further role in the study design; in the collection, analysis and interpretation of data; in the writing of the report; and in the decision to submit the paper for publication.

\section{Conflict of interest}

This work has no conflict of interest.

\section{Acknowledgments}

This research was supported by FEDER Funds through the Programa Operacional Factores de Competitividade - COMPETE and by National Funds through FCT - Fundação para a Ciência e a Tecnologia under the project: PTDC/SAU/SAP/ $116738 / 2010$

\section{References}

Adedinsewo, D.A., Fleming, A.S., Steiner, M., Meaney, M.J., Girard, A.W., MAVAN team, 2014. Maternal anxiety and breastfeeding: findings from the MAVAN (maternal adversity, vulnerability and neurodevelopment) study. J. Hum. Lact. 30, 102-109.

Akman, I., Kuscu, M.K., Yurdakul, Z., Ozdemir, N., Solakoğlu, M., Orhon, L., Karabekiroğlu, A., Ozek, E., 2008. Breastfeeding duration and postpartum psychological adjustment: role of maternal attachment styles. J. Paediatr. Child Health 44, 369-373.

Alder, E.M., Cox, J.L., 1983. Breast feeding and post-natal depression. J. Psychosom. Res. 27, 139-144.

Ali, N.S., Ali, B.S., Azam, I.S., 2009. Postpartum anxiety and depression in peri-urban communities of Karachi, Pakistan: a quasi-experimental study BMC Public Health 9, 384.

American Academy of Pediatrics, 2012. Breastfeeding and the use of human milk. Pediatrics 129, e827-e841.

Annagür, A., Annagür, B.B., Şahin, A., Örs, R., Kara, F., 2013. Is maternal depressive symptomatology effective on success of exclusive breastfeeding during postpartum 6 weeks? Breastfeed. Med. 8, 53-57.

Astbury, J., Brown, S., Lumley, J., Small, R., 1994. Birth events, birth experiences and social differences in postnatal depression. Aust. J. Public Health 18, 176-184.

Austin, M.P.V., Hadzi-Pavlovic, D., Priest, S.R., Reilly, N., Wilhelm, K., Saint, K., Parker, G., 2010. Depressive and anxiety disorders in the postpartum period: how prevalent are they and can we improve their detection? Arch. Women's Ment. Health 13, 395-401.

Bartick, M., Reinhold, A., 2010. The burden of suboptimal breastfeeding in the United States: a pediatric cost analysis. Pediatrics 125, e1048-e1056.

Bick, D.E., MacArthur, C., Lancashire, R.J., 1998. What influences the uptake and early cessation of breast feeding? Midwifery 14, 242-247.

Bogen, D.L., Hanusa, B.H., Moses-Kolko, E., Wisner, K.L., 2010. Are maternal depression or symptom severity associated with breastfeeding intention or outcomes? J. Clin. Psychiatry 71, 1069-1078.

Brion, M.J.A., Lawlor, D.A., Matijasevich, A., Horta, B., Anselmi, L., Araújo, C.L., Menezes, A.M.B., Victora, C.G., Smith, G.D., 2011. What are the causal effects of breastfeeding on IQ obesity and blood pressure? Evidence from comparing high-income with middle-income cohorts. Int. J. Epidemiol. 40, 670-680.

Cattaneo, A., Yngve, A., Koletzko, B., Guzman, L.R., 2005. Protection, promotion and support of breast-feeding in Europe: current situation. Public Health Nutr. 8, 39-46.

Chalmers, B., Heaman, M., Sauve, R., Kaczorowski, J., O’Brien, B., Sauve, R., Kaczorowski, J., 2009. Breastfeeding rates and hospital breastfeeding practices in Canada: a national survey of women. Birth 36, 122-132.

Chaudron, L.H., Klein, M.H., Remington, P., Palta, M., Allen, C., Essex, M.J., 2001. Predictors, prodromes and incidence of postpartum depression. J. Psychosom. Obstet. Gynaecol. 22, 103-112.

Chung, E.K., McCollum, K.F., Elo, I.T., Lee, H.J., Culhane, J.F., 2004. Maternal depressive symptoms and infant health practices among low-income women. Pediatrics 113, 523-529.

Cooper, P.J., Murray, L., Stein, A., 1993. Psychosocial factors associated with the early termination of breast-feeding. J. Psychosom. Res. 37, 171-176.

Dennis, C.L., McQueen, K., 2007. Does maternal postpartum depressive symptomatology influence infant feeding outcomes? Acta Paediatr. 96, 590-594.

Duijts, L., Jaddoe, V.W., Hofman, A., Moll, H.A., 2010. Prolonged and exclusive breastfeeding reduces the risk of infectious diseases in infancy. Pediatrics 126, e18-e25.

Dunn, S., Davies, B., McCleary, L., Edwards, N., Gaboury, I., 2006. The relationship between vulnerability factors and breastfeeding outcomes. J. Obstet. Gynecol. Neonatal Nurs. 35, 87-97.

Ebina, S., Kashiwakura, I., 2012. Influence of breastfeeding on maternal blood pressure at one month postpartum. Int. J. Women's Health 4, 333-339.

Imbula, E.B., Okitundu, L.E., Mampunza, M.M.S., 2012. Postpartum depression in Kinshasa (DR Congo): prevalence and risk factors. Med. Sante Trop. 22, 379-384.

EU Project on Promotion of Breastfeeding in Europe, 2008. Promoting, Protecting and Supporting Breastfeeding: A Blueprint for Action (Revised). European Commission Directorate Public Health, Luxembourg.

Fairlie, T.G., Gilman, M.W., Rich-Edwards, J., 2009. High pregnancy-related anxiety and prenatal depressive symptoms as predictors of intention to breastfeed and breastfeeding initiation. J. Women's Health 18, 945-953.

Feldens, C.A., Vitolo, M.A., Rauber, F., Cruz, L.N., Hilgert, J.B., 2011. Risk factors for discontinuing breastfeeding in southern Brazil: a survival analysis. Matern. Child Health J. 16, 1257-1265.

Field, T., Hernandez-Reif, M., Feijo, M., 2002. Breastfeeding in depressed motherinfant dyads. Early Child Dev. Care 172, 539-545.

Figueiredo, B., Canário, C., Field, T., 2014. Breastfeeding is negatively affected by prenatal depression and reduces postpartum depression. Psychol. Med. 43, $1-10$.

Figueiredo, B., Conde, A., 2011. Anxiety and depression in women and men from early pregnancy to 3-months postpartum. Arch. Women's Ment. Health 14, 247-255.

Figueiredo, B., Pacheco, A., Costa, R., 2007. Depression during pregnancy and the postpartum period in adolescent and adult Portuguese mothers. Arch. Women's Ment. Health 10, 103-109. 
Figueiredo, B., Pacheco, A., Costa, R., Conde, A., Teixeira, C., 2010. Mother's anxiety and depression during the third pregnancy trimester and neonate's mother versus stranger's face/voice visual preference. Early Hum. Dev. 86, 479-485.

Flores-Quijano, M.E., Córdova, A., Contreras-Ramírez, V., Farias-Hernández, L., Cruz Tolentino, M., Casanueva, E., 2008. Risk for postpartum depression, breastfeeding practices, and mammary gland permeability. J. Hum. Lact. 24, 50-57.

Gagliardi, L., Petrozzi, A., Rusconi, F., 2012. Symptoms of maternal depression immediately after delivery predict unsuccessful breast feeding. Arch. Dis. Child. 97, 355-357.

Galler, J.R., Harrisson, R.H., Biggs, M.A., Ramsey, F., Forde, V., 1999. Maternal moods predict breastfeeding in Barbados. J. Dev. Behav. Pediatr. 20, 80-87.

Galler, J.R., Harrison, R.H., Ramsey, F., Chawla, S., Taylor, J., 2006. Postpartum feeding attitudes, maternal depression, and breastfeeding in Barbados. Infant Behav. Dev. 29, 189-203.

González-Jiménez, E., García, P.A., Aguilar, M.J., Padilla, C.A., Álvarez, J., 2013. Breastfeeding and the prevention of breast cancer: a retrospective review of clinical histories. J. Clin. Nurs. 23, 2397-2403.

Groer, M.W., Morgan, K., 2007. Immune, health and endocrine characteristics of depressed postpartum mothers. Psychoneuroendocrinology 32, 133-139.

Haga, S.M, Ullerber, P., Slinning, K., Kraft, P., Steen, T.B., Staff, A., 2012. A longitudinal study of postpartum depressive symptoms: multilevel growth curve analyses of emotion regulation strategies, breastfeeding self-efficacy, and social support. Arch. Women's Ment. Health 15, 175-184.

Hahn-Holbrook, J., Haselton, M.G., Schetter, C.D., Glynn, L.M., 2013. Does breastfeeding offer protection against maternal depressive symptomatology? A prospective study from pregnancy to 2 years after birth. Arch. Women's Ment. Health 16, 411-422.

Hamdan, A., Tamin, H., 2011. Psychosocial risk and protective factors for postpartum depression in the United Arab Emirates. Arch. Women's Ment. Health $14,125-133$.

Hannah, P., Adams, D., Lee, A., Glover, V., Sandler, M., 1992. Links between early post-partum mood and post-natal depression. Br. J. Psychiatry 160, 777-780.

Hasselmann, M.H., Werneck, G.L., Silva, C.V.C., 2008. Symptoms of postpartum depression and early interruption of exclusive breastfeeding in the first two months of life. Cad. Saude Publica 24, 341-352.

Hatton, D., Harrison-Hohner, J., Coste, S., Dorato, V., Curet, L.B., McCarron, D.A., 2005. Symptoms of postpartum depression and breastfeeding. J. Hum. Lact. 21, 444-449.

Henderson, J., Evans, S., Straton, J., Priest, S., Hagan, R., 2003. Impact of postnatal depression on breastfeeding duration. Birth 30, 175-180.

Horta, B., Bahl, R., Martines, J., Victora, C., 2007. Evidence on the Long-Term Effects of Breastfeeding: Systematic Reviews and Meta-Analyses. World Health Organization, Geneva.

Insaf, T.Z., Fortner, R.T., Pekow, P., Dole, N., Markenson, G., Chasan-Taber, L., 2011 Prenatal stress, anxiety, and depressive symptoms as predictors of intention to breastfeed among Hispanic women. J. Women's Health 20, 1183-1192.

Jonas, W., Nissen, E., Ransjö-Arvidson, A.B., Wiklund, I., Henriksson, P., UvnäsMoberg, K., 2008. Short and long-term decrease of blood pressure in women during breastfeeding. Breastfeed. Med. 3, 103-109.

Kehler, H.L., Chaput, K.H., Tough, S.C., 2009. Risk factors for cessation of breastfeeding prior to six months postpartum among a community sample of women in Calgary, Alberta. Can. J. Public Health 100, 376-380.

Kennerley, H., Gath, D., 1989. Maternity blues: I. Detection and measurement by questionnaire. Br. J. Psychiatry 155, 356-362.

Kumar, R., Robson, K., 1984. A prospective study of emotional disorders in childbearing women. Br. J. Psychiatry 144, 35-47.

Lau, Y., Chan, K.S., 2007. Influence of intimate partner violence during pregnancy and early postpartum depressive symptoms on breastfeeding among Chinese women in Hong Kong. J. Midwifery Women's Health 52, 15-20.

Lee, W.S., Cho, J., Choi, Y.S., Chung, S.H., Bae, C.W., Jung, J.A., 2013. Breastfeeding rate in below 6 months infants during recent 6-year in Korea based on childcare database. Neonatal Med. 20, 221-227.

McCarter-Spaulding, D., Horowitz, J.A., 2007. How does postpartum depression affect breastfeeding? MCN: Am. J. Matern. Child Nurs. 32, 10-17.
McCoy, S.J.B., Beal, J.M., Shipman, S.B.M., Payton, M.E., Watson, G.H., 2006. Risk factors for postpartum depression: a retrospective investigation at 4-weeks postnatal and a review of the literature. J. Am. Osteopath. Assoc. 106, 193-198.

McLearn, K.T., Minkowitz, C.S., Strobino, D.M., Marks, E., Hou, W., 2006. Maternal depressive symptoms at 2 to 4 months postpartum and early parenting practices. Arch. Pediatr. Adolesc. Med. 160, 279-284.

Mezzacappa, E.S., Endicott, J., 2007. Parity mediates the association between infant feeding method and maternal depressive symptoms in the postpartum. Arch. Women's Ment. Health 10, 259-266.

Milgrom, J., Gemmill, A.W., Bilszta, J.L., Hayes, B., Barnett, B., Brooks, J., Ericksen, J., Ellwood, D., Buist, A., 2008. Antenatal risk factors for postnatal depression: a large prospective study. J. Affect. Disord. 108, 147-157.

Misri, S., Sinclair, D.A., Kuan, A.J., 1997. Breast-feeding and postpartum depression: is there a relationship? Can. J. Psychiatry 42, 1061-1065.

Moher, D., Liberatti, A., Tetzlaff, J., Altman, D.G., 2009. PRISMA GroupPreferred reporting items for systematic reviews and meta-analysis: the prisma statement. Ann. Intern. Med. 151, 264-269.

Murray, L., Cooper, P.J., 1997. Postpartum Depression and Child Development. The Guilford Press, NY, pp. 3-34.

Nishioka, E. Haruna, M. Ota, E., Matsuzaki, M., Murayama, R., Yoshimura, K. Murashima, S., 2011. A prospective study of the relationship between breastfeeding and postpartum depressive symptoms appearing at 1-5 months after delivery. J. Affect. Disord. 133, 553-559.

O'Hara, M.W., 1997. The nature of postpartum affective disorders. In: Murray, L. Cooper, P.J. (Eds.), Postpartum Depression and Child Development. The Guilford Press, NY, pp. 3-34.

Papinczak, T.A., Turner, C.T., 2000. An analysis of personal and social factors influencing initiation and duration of breastfeeding in a large Queensland maternity hospital. Breastfeed. Rev. 8, 25-33.

Paul, I.M., Downs, D.S., Schaefer, E.W., Beiler, J.S., Weisman, C.S., 2013. Postpartum anxiety and maternal-infant health outcomes. Pediatrics 131, e1218-e1224.

Pippins, J.R., Brawarsky, P., Jackson, R.A., Fuentes-Afflick, E., Haas, J.S., 2006 Association of breastfeeding with maternal depressive symptom. J. Women's Health 15, 754-762.

Pitt, B., 1968. Atypical depression following childbirth. Br. J. Psychiatry 114, 1325-1335.

Seimyr, L., Edhborg, M., Lundhand, W., Sjögren, B., 2004. In the shadow of maternal depressed mood: experiences of parenthood during the first year after childbirth. J. Psychosom. Obstetr. Gynecol. 25, 23-34.

Taj, R., Sikander, K.S., 2003. Effects of maternal depression on breast-feeding. J. Pak. Med. Assoc. 53, 8-11.

Tamminen, T., 1988. The impact of mother's depression on her nursing experiences and attitudes during breastfeeding. Acta Paediatr. Scand. Suppl. 344, 87-94.

Taveras, E., Capra, A., Braveman, P., Jensvold, N., Escobar, G., Lieu, T., 2003. Clinician support and psychosocial risk factors associated with breastfeeding discontinuation. Pediatrics 112, 108-115.

Thome, M., Alder, E.M., Ramel, A., 2006. A population-based study of exclusive breastfeeding in Icelandic women: is there a relationship with depressive symptoms and parenting stress? Int. J. Nurs. Stud. 43, 11-20.

Watkins, S., Meltzer-Brody, S., Zolnoun, D., Stuebe, A., 2011. Early breastfeeding experiences and postpartum depression. Obstetr. Gynecol. 11, 214-221.

World Health Organization, 2007. Planning Guide for National Implementation of the Global Strategy for Infant and Young Child Feeding. World Health Organization, Geneva.

Yalom, I.D., Lunde, D.T., Moos, R.H., Hamburg, D.A., 1968. Postpartum blues syndrome. A description and related variables. Arch. Gen. Psychiatry 18, 16-27.

Yonkers, K.A., Ramin, S., Rush, A.J., Navarrete, C.A., Carmody, T., March, D., Heatwell, S.F., Leveno, K.J., 2001. Onset and persistence of postpartum depression in an inner-city maternal health clinic system. Am. J. Psychiatry 158, 1856-1863.

Ystrom, E., 2012. Breastfeeding cessation and symptoms of anxiety and depression: a longitudinal cohort study. BMC Pregnancy Childbirth 12, 12-36.

Zubaran, C., Foresti, K., 2013. The correlation between breastfeeding self-efficacy and maternal postpartum depression in southern Brazil. Sex Reprod. Healthc. 4, 9-15. 